\title{
A Patch-Based Low-Rank Minimization Approach for Speckle Noise Reduction in Ultrasound Images
}

\author{
Xiao-Guang $\mathrm{Lv}^{1}$, Fang $\mathrm{Li}^{2}{ }^{2}$, Jun $\mathrm{Liu}^{3}$ and Sheng-Tai $\mathrm{Lu}^{1}$ \\ ${ }^{1}$ School of Science, Jiangsu Ocean University, Lianyungang, Jiangsu 222005, China \\ 2 Shanghai Key Laboratory of PMMP and School of Mathematical Sciences, East China \\ Normal University, Shanghai 200241, China \\ 3 School of Mathematics and Statistics and Key Laboratory of Applied Statistics of MOE, \\ Northeast Normal University, Changchun, Jilin 130024, China
}

Received 17 January 2021; Accepted (in revised version) 31 March 2021

\begin{abstract}
Ultrasound is a low-cost, non-invasive and real-time imaging modality that has proved popular for many medical applications. Unfortunately, the acquired ultrasound images are often corrupted by speckle noise from scatterers smaller than ultrasound beam wavelength. The signal-dependent speckle noise makes visual observation difficult. In this paper, we propose a patch-based low-rank approach for reducing the speckle noise in ultrasound images. After constructing the patch group of the ultrasound images by the block-matching scheme, we establish a variational model using the weighted nuclear norm as a regularizer for the patch group. The alternating direction method of multipliers (ADMM) is applied for solving the established nonconvex model. We return all the approximate patches to their original locations and get the final restored ultrasound images. Experimental results are given to demonstrate that the proposed method outperforms some existing state-of-the-art methods in terms of visual quality and quantitative measures.
\end{abstract}

AMS subject classifications: 68U10, 15A29, 65K05

Key words: Ultrasound images, patch, speckle noise, low-rank, weighted nuclear norm minimization.

\section{Introduction}

Ultrasound images provide the clinician with low-cost, non-invasive, and real-time images of the internal structure of the body that can help them detect deadly diseases or abnormal tissues [1-4]. As a portable and fast imaging modality, ultrasound has proved

*Corresponding author.

Emails: xiaoguanglv@jou.edu.cn (X-G. Lv), fli@math.ecnu.edu.cn (F. Li), liuj292@nenu.edu.cn (J. Liu), lushengtai@126.com (S-T. Lu) 
popular for many medical applications [5-8]. However, ultrasound images usually suffer from the signal-dependent speckle noise, limiting the contrast resolution and making it generally difficult for human interpretation and diagnosis. Therefore, speckle noise reduction is an important issue in medical ultrasound image processing $[9,10]$.

The ultrasound images corrupted with the signal-dependent noise can be modeled as the following form:

$$
g=u+\sqrt{u} \eta,
$$

where $g$ is the corrupted ultrasound image, $u$ is the original image, $\eta$ is a zero-mean Gaussian noise with standard deviation $\delta$. To restore $u$ from the corrupted ultrasound image $g$ is the main task of speckle noise reduction.

Several variational methods have been proposed to reduce the speckle noise in ultrasound images in recent decades [11]. In [12], Jin and Yang studied the following total variation based model for dealing with the speckle noise in ultrasound images:

$$
\min _{u} \int_{\Omega} \frac{(u-g)^{2}}{u} d x+\lambda \int_{\Omega}|\nabla u| d x,
$$

where $\Omega \subset \mathbb{R}^{2}$ is a bounded set, $\lambda$ is the regularization parameter for measuring the tradeoff between the fidelity term and the regularized term, and the regularization term is the total variation of $u$. They proved the existence and uniqueness of the minimizer for the variational problem and derived the existence and uniqueness of weak solutions for the associated evolution equation. In [13], Huang and Yang proposed a convex variational model to deal with the speckle noise in real ultrasound images where the data-fitting term is the generalized Kullback-Leibler distance and the regularization term is the total variation of the estimated image.

It is widely known that the classic total variation based model usually suffers from the staircase effect. Some high-order total variation, total generalized variation, and hybrid regularization based models are proposed to overcome this shortcoming and preserve edges in the restored images well [14,15]. In [16], Wang et al. proposed a variation model for speckle noise removal in ultrasound images where the regularization term is represented by a combination of total variation and high-order total variation (HTV) and the data fidelity term is depicted by a generalized Kullback-Leibler divergence. They solved the first-order and second-order total variation based model using the alternating direction method with multipliers (ADMM). In [17], Mei et al. investigated a second-order total generalized variation regularization to solve the problem of speckle noise reduction in ultrasound images. Numerical experiments have shown that the second-order total generalized variation based model outperforms some state-of-the-art methods in terms of visual quality, peak signal-to-noise ration (PSNR) and structural similarity (SSIM) index. In [18], Abrahim and Kadah presented a combined technique using wavelet shrinkage and total variation for speckle noise removal in clinical ultrasound images. The hybrid method takes full advantage of total variation based method to denoise the low frequency subbands without losing textures, and uses the wavelet shrinkage method based on local variance information to extract textures from noise in the high frequency subbands. 
Although the variational methods have many desirable properties, they fail to consider the structural sparsity of images. Usually, a local patch of natural images has many similar patches to it. In recent years, image restoration using the similarity of nonlocal patches for exploiting the structural sparsity of natural images has drawn much attention [19-23]. The patch-based methods succeed in suppressing most of the noise with good preservation of texture and removal of the coherent aliasing artifacts. A typical example is the so-called block matching and 3D filtering (BM3D) denoising method [24]. The BM3D method achieves remarkable results by applying the collaborative filtering in the transformed domain on the stack of nonlocal similar patches. The low-rank approximation of the patch group has been studied and applied to image denoising in [25]. They used the weighted nuclear norm term as the self-similarity prior for the patch group and solved the weighted nuclear norm minimization (WNNM) problem with the soft thresholding operator. Experimental results were given to demonstrate that the WNNM method can achieve better performance than some state-of-the-art denoising methods.

Motivated by the idea of the patch-based methods, we use a patch-based low-rank minimization approach for speckle noise reduction in ultrasound images. Firstly, we divide the whole noisy image into several overlapped key patches and construct the patch group for each key patch by the block matching technique. Secondly, we propose a lowrank minimization model for the patch groups based on the weighted nuclear norm. We employ the ADMM for solving the proposed low-rank minimization model and get the low-rank approximation of all patch groups. We give the weak convergence result of the nonconvex ADMM. At last, we aggregate all the resulting patches for getting the whole estimated image. Numerical experiments are given to show that the proposed method outperforms some existing state-of-the-art methods in terms of visual quality and quantitative measures.

The main contributions of this paper are summarized as follows:

(i) We use the patch-based low-rank minimization approach for speckle noise reduction in ultrasound images. The proposed patch-based low-rank minimization method makes full use of the distinctive structural characteristics of the processed images. It can reduce the speckle noise in ultrasound images very well.

(ii) We use the ADMM to solve the proposed patch-based low-rank minimization problem by introducing an auxiliary variable. The resulting subproblems are simple and easy to be solved. The first subproblem can be solved by the Newton's method. The second subproblem has a closed-form solution.

(iii) We show the numerical experiments on simulated images and real ultrasound images. It can be seen from the experiments that the proposed method outperforms some existing state-of-the-art methods in terms of visual quality and quantitative measures. 


\section{Related work}

In this section, we recall some results about the WNNM model and briefly describe the block matching technique.

\subsection{WNNM}

The low-rank term has been emerged as an important prior knowledge of the processed images. Methods based on the weighted nuclear norm term have been widely studied in image processing [25-28]. The weighted nuclear norm of a matrix $X$ is defined as a weighted sum of its singular value:

$$
\|X\|_{w, *}=\sum_{j=1}^{n} w_{j} \sigma_{j}(X)
$$

where $w=\left(w_{1}, \cdots, w_{n}\right)^{T}, \sigma_{j}(X)$ means the $j$ th singular value of matrix $X, w_{j} \geq 0$ is a weight assigned to the $j$ th singular value and $n$ is the number of singular values of matrix $X$. In [25], Gu et al. have proposed the following WNNM model for image denoising:

$$
\min _{X}\|X\|_{w, *}+\frac{1}{2}\|X-Y\|_{F}^{2}
$$

where $\|\cdot\|_{F}$ is the Frobenius norm. Let $Y=U \Sigma V^{T}$ be the singular value decomposition of matrix $Y$. Then the solution $\hat{X}$ of the optimization problem (2.1) can be expressed as $\hat{X}=U \hat{\Lambda} V^{T}$, where $\hat{\Lambda}$ is the solution of the following minimization problem:

$$
\min _{\Lambda}\|\Lambda\|_{w, *}+\frac{1}{2}\|\Lambda-\Sigma\|_{F}^{2}
$$

Especially, if the weights are in a non-descending order $\left(0 \leq w_{1} \leq \cdots \leq w_{n}\right)$, the optimal solution of the WNNM problem (2.1) is $\hat{X}=U \hat{\Lambda} V^{T}$ where the diagonal matrix $\hat{\Lambda}=\mathcal{S}_{w}(\Sigma)$ with $\mathcal{S}_{w}(\Sigma)_{j j}=\max \left(\Sigma_{j j}-w_{j}, 0\right)$ for all $j$.

\subsection{Block matching}

The success of any patch-based noise reduction method relies greatly on the ability to find similar patches in the noisy image and operate on the gathering patch groups [24,29, 30]. The goal of block-matching is to gather a group of image patches containing similar image structures. Usually, the block-matching procedure contains three steps. At the first step, divide the noisy image into overlapped patches of relatively small size and choose some appropriate image patches as the key patches. For each key patch $y$, consider all the overlapped patches contained in its nonlocal neighborhood. At the second step, find the patch $y_{i}$ similar to each given key patch in the nonlocal neighborhood. The Euclidean distance $\left\|y-y_{i}\right\|_{F}$ is used to measure the similarity between the key patch and the patch 


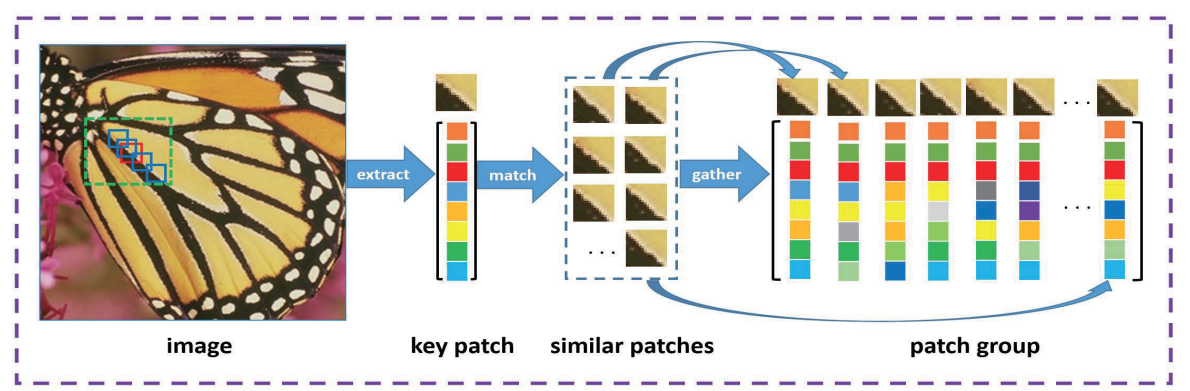

Figure 1: Overview of block matching.

extracted from the nonlocal neighborhood. A smaller distance indicates higher similarity. At the last step, choose the $K$ most similar patches to the key patch among the patches contained in the nonlocal neighborhood. By stacking the columns of the $K$ most similar patches, construct the patch group matrix $Y$. In Fig. 1, we show the overall process of the block matching technique. For the sake of a clear presentation, we take a color image for example in Fig. 1.

\section{The proposed model and algorithm}

We use the patch-based low-rank minimization approach for speckle noise reduction in ultrasound images. The proposed patch-based low-rank method contains three steps: (i) Extract the key patches and construct the patch group for each key patch by the blockmatching scheme; (ii) For each patch group, establish the variational low-rank model by using the weighted nuclear norm as a regularizer and solve the proposed variational model with the ADMM; (iii) Compute the final estimated ultrasound image by averaging all approximate patch groups.

The core of the proposed patch-based method is to operate the patch-based low-rank minimization on the patch groups. Let $Y$ be any patch group of the input noisy image. For the patch group $Y$, we want to solve the following optimization problem:

$$
\min _{X} \sum_{i, j} \frac{\left(X_{i, j}-Y_{i, j}\right)^{2}}{X_{i, j}}+\|X\|_{w, *} .
$$

The splitting iteration algorithms such as ADMM, split-Bregman algorithm and modulus splitting iteration $[31,32]$ can be employed to solve the proposed minimization problem (3.1). In this paper, we apply the effective ADMM for solving the nonconvex patch group minimization problem (3.1). By introducing an auxiliary variable $Z$, the problem (3.1) can be reformulated as the following constrained optimization problem:

$$
\min _{X, Z} \sum_{i, j} \frac{\left(X_{i, j}-Y_{i, j}\right)^{2}}{X_{i, j}}+\|Z\|_{w, *}, \quad \text { s.t. } X=Z \text {. }
$$


The augmented Lagrangian function of (3.2) can be written as the following form:

$$
\mathcal{L}(X, Z, W, \rho)=\sum_{i, j} \frac{\left(X_{i, j}-Y_{i, j}\right)^{2}}{X_{i, j}}+\|Z\|_{w, *}+\langle W, X-Z\rangle+\frac{\rho}{2}\|X-Z\|_{F}^{2},
$$

where $W$ is the Lagrange multiplier and $\rho$ is a penalty parameter. The idea of the ADMM is to solve the following subproblems iteratively:

$$
\left\{\begin{array}{l}
X^{k+1}=\operatorname{argmin}_{X} \mathcal{L}\left(X, Z^{k}, W^{k}, \rho^{k}\right) \\
Z^{k+1}=\operatorname{argmin}_{Z} \mathcal{L}\left(X^{k+1}, Z, W^{k}, \rho^{k}\right), \\
W^{k+1}=W^{k}+\rho^{k}\left(X^{k+1}-Z^{k+1}\right) \\
\rho^{k+1}=\mu \rho^{k}
\end{array}\right.
$$

where $\mu>1$ is a given parameter.

To minimize $\mathcal{L}$ with respect to $X$, we solve the subproblem

$$
\min _{X} \sum_{i, j} \frac{\left(X_{i, j}-Y_{i, j}\right)^{2}}{X_{i, j}}+\frac{\rho^{k}}{2}\left\|X-Z^{k}+\frac{1}{\rho^{k}} W^{k}\right\|_{F}^{2} .
$$

It is obvious that the minimizations with respect to $X$ can be solved separately, since they are decoupled. Hence, the minimizer of the problem (3.5) is equivalent to solving the following nonlinear systems:

$$
1-\frac{Y_{i, j}^{2}}{X_{i, j}^{2}}+\rho^{k}\left(X_{i, j}-Z_{i, j}^{k}\right)+W_{i, j}^{k}=0 .
$$

The solution of (3.6) can be determined very efficiently by using the Newton's method.

As for $Z$, the subproblem is

$$
\min _{Z}\|Z\|_{w, *}+\frac{\rho^{k}}{2}\left\|Z-\left(X^{k+1}+\frac{1}{\rho^{k}} W^{k}\right)\right\|_{F}^{2}
$$

Suppose that

$$
X^{k+1}+\frac{1}{\rho^{k}} W^{k}=U^{k} \Sigma^{k}\left(V^{k}\right)^{T}
$$

is the singular value decomposition of $X^{k+1}+\frac{1}{\rho^{k}} W^{k}$. For the weights in a non-descending order, the closed-form solution of the problem (3.7) is given by

$$
Z^{k+1}=U^{k} \mathcal{S}_{w / \rho^{k}}\left(\Sigma^{k}\right)\left(V^{k}\right)^{T},
$$

where

$$
\mathcal{S}_{w / \rho^{k}}\left(\Sigma^{k}\right)_{j j}=\max \left(\sum_{j j}^{k}-w_{j} / \rho^{k}, 0\right)
$$




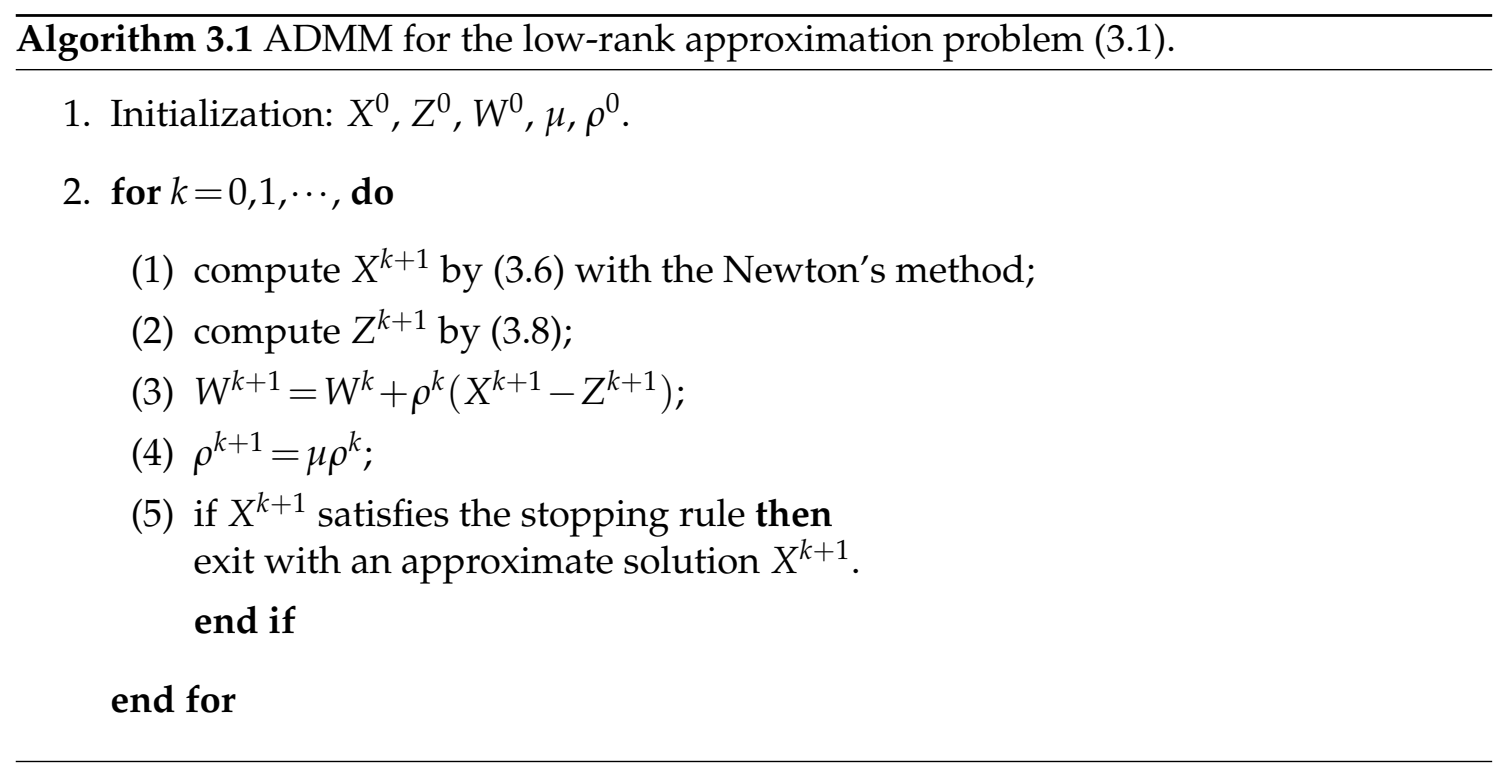

for all $j$.

To sum up, we obtain the following algorithm for solving the patch group minimization problem.

There are many convergence results for ADMM discussed in the literature [32-34]. However, the convergence of Algorithm 3.1 is difficult to analyze due to the nonconvexity of the weighted nuclear norm. We give the following weak convergence result to facilitate the construction of a rational termination condition for Algorithm 3.1.

Theorem 3.1. If the weights are sorted in a nondescending order, the sequences $\left\{X^{k}\right\},\left\{U^{k}\right\}$ and $\left\{Z^{k}\right\}$ generated by Algorithm 3.1 satisfy:

$$
\begin{aligned}
& \text { (i) } \lim _{k \rightarrow \infty}\left\|X^{k+1}-Z^{k+1}\right\|_{F}=0, \\
& \text { (ii) } \lim _{k \rightarrow \infty}\left\|X^{k+1}-X^{k}\right\|_{F}=0, \\
& \text { (iii) } \lim _{k \rightarrow \infty}\left\|Z^{k+1}-Z^{k}\right\|_{F}=0
\end{aligned}
$$

Proof. (i) Suppose that

$$
X^{k+1}+\frac{1}{\rho^{k}} W^{k}=U^{k} \Sigma^{k}\left(V^{k}\right)^{T}
$$

is the singular value decomposition of $X^{k+1}+\frac{1}{\rho^{k}} W^{k}$. Then we have

$$
Z^{k+1}=U^{k} \mathcal{S}_{w / \rho^{k}}(\Sigma)\left(V^{k}\right)^{T},
$$

where

$$
\mathcal{S}_{w / \rho^{k}}\left(\Sigma^{k}\right)_{j j}=\max \left(\Sigma_{j j}^{k}-w_{j} / \rho^{k}, 0\right)
$$


for all $j$. Firstly, we show the bounded property of $\left\{W^{k}\right\}$

$$
\begin{aligned}
\left\|W^{k+1}\right\|_{F} & =\left\|W^{k}+\rho^{k}\left(X^{k+1}-Z^{k+1}\right)\right\|_{F} \\
& \left.=\rho^{k} \| X^{k+1}+\frac{1}{\rho^{k}} W^{k}-Z^{k+1}\right) \|_{F} \\
& =\rho^{k}\left\|U^{k}\left(\mathcal{S}_{w / \rho^{k}}\left(\Sigma^{k}\right)-\Sigma^{k}\right)\left(V^{k}\right)^{T}\right\|_{F} \\
& =\rho^{k}\left\|\left(\mathcal{S}_{w / \rho^{k}}\left(\Sigma^{k}\right)-\Sigma^{k}\right)\right\|_{F} \\
& \leq \rho^{k}\left\|w / \rho^{k}\right\|_{2}=\|w\|_{2} .
\end{aligned}
$$

It is obvious that

$$
\mathcal{L}\left(X^{k+1}, Z^{k+1}, W^{k}, \rho^{k}\right) \leq \mathcal{L}\left(X^{k+1}, Z^{k}, W^{k}, \rho^{k}\right) \leq \mathcal{L}\left(X^{k}, Z^{k}, W^{k}, \rho^{k}\right) .
$$

By straightforward calculations, we have the following equality:

$$
\begin{aligned}
& \mathcal{L}\left(X^{k}, Z^{k}, W^{k}, \rho^{k}\right)-\mathcal{L}\left(X^{k}, Z^{k}, W^{k-1}, \rho^{k-1}\right) \\
= & \left\langle W^{k}-W^{k-1}, X^{k}-Z^{k}\right\rangle+\frac{\rho^{k}-\rho^{k-1}}{2}\left\|X^{k}-Z^{k}\right\|_{F} \\
= & \frac{\rho^{k}+\rho^{k-1}}{2\left(\rho^{k-1}\right)^{2}}\left\|W^{k}-W^{k-1}\right\|_{F} .
\end{aligned}
$$

Thus, we may immediately obtain that

$$
\mathcal{L}\left(X^{k+1}, Z^{k+1}, W^{k}, \rho^{k}\right) \leq \mathcal{L}\left(X^{k}, Z^{k}, W^{k-1}, \rho^{k-1}\right)+\frac{\rho^{k}+\rho^{k-1}}{2\left(\rho^{k-1}\right)^{2}}\left\|W^{k}-W^{k-1}\right\|_{F} .
$$

We denote by $M$ the upper bound of $\left\|W^{k}-W^{k-1}\right\|_{F}$ for all $k$, since $\left\{W^{k}\right\}$ is bounded. Hence, we have

$$
\mathcal{L}\left(X^{k+1}, Z^{k+1}, W^{k}, \rho^{k}\right) \leq \mathcal{L}\left(X^{1}, Z^{1}, W^{0}, \rho^{0}\right)+M \sum_{k}^{\infty} \frac{\rho^{k}+\rho^{k-1}}{2\left(\rho^{k-1}\right)^{2}} .
$$

Since $\rho^{k+1}=\mu \rho^{k}$, we obtain that

$$
M \sum_{k}^{\infty} \frac{\rho^{k}+\rho^{k-1}}{2\left(\rho^{k-1}\right)^{2}}=\frac{M \mu(\mu+1)}{2 \rho^{0}} \sum_{k}^{\infty} \mu^{-k}<\infty
$$

Therefore, $\mathcal{L}\left(X^{k+1}, Z^{k+1}, W^{k}, \rho^{k}\right)$ is upper bounded. The boundedness of $\left\{X^{k}\right\}$ and $\left\{Z^{k}\right\}$ can be easily deduced by

$$
\begin{aligned}
& \mathcal{L}\left(X^{k+1}, Z^{k+1}, W^{k}, \rho^{k}\right)+\frac{1}{2 \rho^{k}}\left\|W^{k}\right\|_{F}^{2} \\
= & \sum_{i, j} \frac{\left(X_{i, j}^{k+1}-Y_{i, j}\right)^{2}}{X_{i, j}^{k+1}}+\left\|Z^{k+1}\right\|_{w, *}+\frac{\rho^{k}}{2}\left\|X^{k+1}-Z^{k+1}+\frac{1}{\rho^{k}} W^{k}\right\|_{F}^{2} .
\end{aligned}
$$


Based on the fact that $\rho^{k} \rightarrow \infty$ as $k \rightarrow \infty$, we get

$$
\lim _{k \rightarrow \infty}\left\|X^{k+1}-Z^{k+1}\right\|_{F}=\lim _{k \rightarrow \infty} \frac{1}{\rho^{k}}\left\|W^{k+1}-W^{k}\right\|_{F}=0 .
$$

(ii) Consider the following optimization problem:

$$
X^{k+1}=\arg \min _{X} f(X)+\frac{\rho^{k}}{2}\left\|X-Z^{k}+\frac{1}{\rho^{k}} W^{k}\right\|_{F^{\prime}}^{2}
$$

where

$$
f(X)=\sum_{i, j} \frac{\left(X_{i, j}-Y_{i, j}\right)^{2}}{X_{i, j}} .
$$

The first-order optimality of this problem is given by

$$
-\frac{\nabla f\left(X^{k+1}\right)}{\rho^{k}}=X^{k+1}-Z^{k}+\frac{1}{\rho^{k}} W^{k}
$$

According to $W^{k}=W^{k-1}+\rho^{k-1}\left(X^{k}-Z^{k}\right)$, we may obtain

$$
X^{k+1}-X^{k}=-\frac{\nabla f\left(X^{k+1}\right)}{\rho^{k}}-\frac{1}{\rho^{k-1}}\left(W^{k}-W^{k-1}\right)-\frac{1}{\rho^{k}} W^{k},
$$

where

$$
\nabla f\left(X^{k+1}\right)=\sum_{i, j}\left(1-\frac{\left(Y_{i, j}^{k+1}\right)^{2}}{\left(X_{i, j}^{k+1}\right)^{2}}\right) .
$$

Numerically, we add a small number $\epsilon>0$ to avoid dividing by zero, that is, we let

$$
\nabla f\left(X^{k+1}\right)=\sum_{i, j}\left(1-\frac{\left(Y_{i, j}^{k+1}\right)^{2}}{\left(X_{i, j}^{k+1}\right)^{2}+\epsilon}\right) .
$$

Hence, $\nabla f\left(X^{k+1}\right)$ is bounded, since $X^{k+1}$ is bounded. Then, we get

$$
\begin{aligned}
\lim _{k \rightarrow \infty}\left\|X^{k+1}-X^{k}\right\|_{F} & =\lim _{k \rightarrow \infty}\left(\left\|\frac{\nabla f\left(X^{k+1}\right)}{\rho^{k}}+\frac{1}{\rho^{k-1}}\left(W^{k}-W^{k-1}\right)+\frac{1}{\rho^{k}} W^{k}\right\|_{F}\right) \\
& \leq \lim _{k \rightarrow \infty}\left(\frac{\left\|\nabla f\left(X^{k+1}\right)\right\|_{F}}{\rho^{k}}+\frac{\left\|W^{k}-W^{k-1}\right\|_{F}}{\rho^{k-1}}+\frac{\left\|W^{k}\right\|_{F}}{\rho^{k}}\right)=0 .
\end{aligned}
$$


(iii) Based on the updating rule of $X$ and $W$, we have

$$
\begin{aligned}
& \lim _{k \rightarrow \infty}\left\|Z^{k+1}-Z^{k}\right\|_{F}=\lim _{k \rightarrow \infty}\left\|X^{k+1}-\frac{1}{\rho^{k}} W^{k+1}+\frac{1}{\rho^{k}} W^{k}-Z^{k}\right\|_{F} \\
= & \lim _{k \rightarrow \infty}\left\|\left(X^{k+1}-X^{k}\right)+\left(X^{k}+\frac{1}{\rho^{k-1}} W^{k-1}-Z^{k}\right)+\left(\frac{1}{\rho^{k}} W^{k}-\frac{1}{\rho^{k-1}} W^{k-1}-\frac{1}{\rho^{k}} W^{k+1}\right)\right\|_{F} \\
\leq & \lim _{k \rightarrow \infty}\left(\left\|X^{k+1}-X^{k}\right\|_{F}+\left\|X^{k}+\frac{1}{\rho^{k-1}} W^{k-1}-Z^{k}\right\|_{F}+\left\|\frac{1}{\rho^{k}} W^{k}-\frac{1}{\rho^{k-1}} W^{k-1}-\frac{1}{\rho^{k}} W^{k+1}\right\|_{F}\right) \\
\leq & \lim _{k \rightarrow \infty}\left\|X^{k}+\frac{1}{\rho^{k-1}} W^{k-1}-Z^{k}\right\|_{F}=\lim _{k \rightarrow \infty}\left\|U^{k-1}\left(\mathcal{S}_{w / \rho^{k-1}}\left(\Sigma^{k-1}\right)-\Sigma^{k}\right)\left(V^{k-1}\right)^{T}\right\|_{F} \\
= & \left.\lim _{k \rightarrow \infty} \|\left(\mathcal{S}_{w / \rho^{k-1}}\left(\Sigma^{k-1}\right)-\Sigma^{k}\right)\right)\left\|_{F} \leq \lim _{k \rightarrow \infty} \frac{1}{\rho^{k-1}}\right\| w \|_{2}=0 .
\end{aligned}
$$

Thus, we complete the proof.

By Algorithm 3.1, we may compute the low-rank approximation of all key patch group and obtain all approximate patches. Then we return all the approximate patches to their original locations. Since the patches are overlapped, every pixel is finally estimated as the average of repeated estimates. Therefore, we have the following algorithm for speckle noise reduction in ultrasound images.

Algorithm 3.2 Patch-based algorithm for the speckle noise reduction problem.

1. Input: Noisy image $g$.

2. for $k=0,1, \cdots, M$, do

(1) for each key patch $Y$ do

i. Find similar patches and construct the patch group;

ii. Apply Algorithm 3.1 for solving the low-rank approximation problem (3.1).

end for

(2) Aggregate all the resulting approximate patches and form the restored image.

end for

3. Output: Restored image $\tilde{u}$.

In Fig. 2, we show the overall process of the proposed speckle noise reduction algorithm. For the sake of clear presentation, we take a color image for example in Fig. 2. In the inner iteration, we use the ADMM for each key patch low-rank approximation. In the outer iteration, we update the resulting image formed by the inner iteration. When 


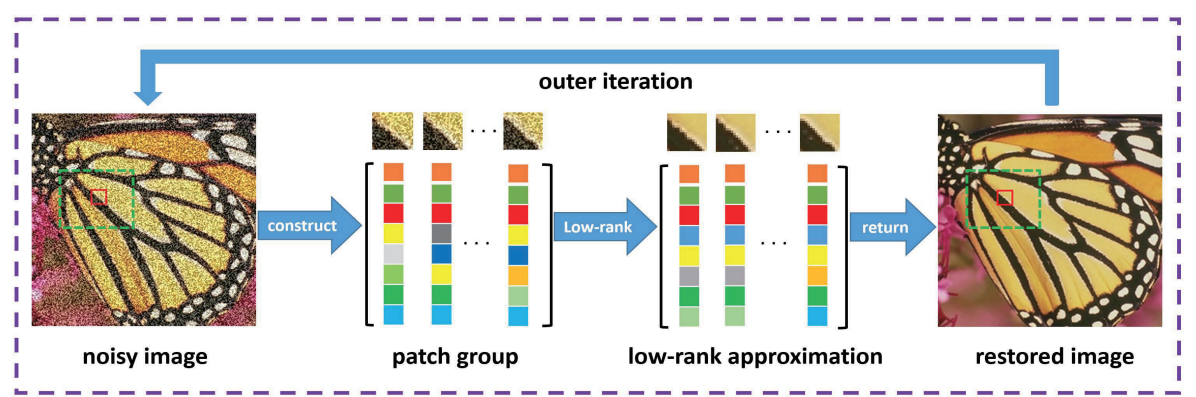

Figure 2: Overview of the proposed speckle noise reduction algorithm.

the maximum outer iteration number is reached or the relative error between two successive outer iterations satisfies the stopping criterion, we get the final approximate restored image.

\section{Numerical experiments}

In this section, we give some numerical experiments to illustrate the effectiveness of the proposed patch-based low-rank minimization method for speckle noise reduction in ultrasound images. We compare the proposed method with some existing speckle noise reduction methods including TV [12], HTV [16], TGV [17] BM3D [29] and denoising convolutional neural network (DnCNN) [35] on both synthetic images and real ultrasound images. All of the numerical experiments were carried out in Matlab R2018a. The results were obtained by running the Matlab codes on an Intel(R) Core(TM) i7-8700 CPU (3.70GHz, 3.70GHz) computer with RAM of 16GB.

To evaluate the quality of the restoration results quantitatively, we use the PSNR and SSIM results. The definition of PSNR is given by

$$
\operatorname{PSNR}=10 \log _{10}\left(\frac{m n \cdot \max _{u}^{2}}{\|\tilde{u}-u\|_{2}^{2}}\right),
$$

where $u$ and $\tilde{u}$ are the $m \times n$ ideal image and the restored image respectively, and $\max _{u}$ is the maximum possible pixel value of the image $u$. For example, when the pixels are represented by 8 bits per sample, the value of $\max _{u}$ is 255 . The SSIM is defined as the mean of local similarity indexes:

$$
\operatorname{SSIM}=\frac{1}{N} \sum_{i=1}^{N} \operatorname{ssim}\left(x_{i}, \tilde{x}_{i}\right)
$$

where

$$
\operatorname{sim}\left(x_{i}, \tilde{x}_{i}\right)=\frac{\left(2 \mu_{x_{i}} \mu_{\tilde{x}_{i}}+C_{1}\right)\left(2 \sigma_{x_{i} \tilde{x}_{i}}+C_{2}\right)}{\left(\mu_{x_{i}}^{2}+\mu_{\tilde{x}_{i}}^{2}+C_{1}\right)\left(\sigma_{x_{i}}^{2}+\sigma_{\tilde{x}_{i}}^{2}+C_{2}\right)} .
$$


Here $x_{i}$ and $\tilde{x}_{i}$ are corresponding windows of $u$ and $\tilde{u}$ indexed by $i$ respectively and $N$ is the number of windows. $\mu_{x_{i}}$ and $\mu_{\tilde{x}_{i}}$ are averages of $x_{i}$ and $\tilde{x}_{i}$, respectively. $\sigma_{x_{i}}$ and $\sigma_{\tilde{x}_{i}}$ are the variances of $x_{i}$ and $\tilde{x}_{i}$, respectively. $\sigma_{x_{i} \tilde{x}_{i}}$ is the covariance of $x_{i}$ and $\tilde{x}_{i}$. The positive constants $C_{1}$ and $C_{2}$ can be thought of as stabilizing constants for near-zero denominator values. We refer the reader to [36] for further details on SSIM. In the experiments, we will use the SSIM value to reveal areas of high or low similarities between two images. It is noted that the closer the SSIM value is to 1, the stronger the structural similarity between the restored image and the original image is.

As is done in [25], we choose the weight for the key patch group by using

$$
w_{j}=\tilde{\delta}^{2} * \min \left\{\frac{C \sqrt{K}}{\sqrt{\max \left\{\sigma_{j}^{2}(Y)-K \tilde{\delta}^{2}, 0\right\}}+\varepsilon}, 1\right\},
$$

where $\tilde{\delta}$ is the noise standard deviation of the patch group, $K$ is the number of patches in a patch group. $\sigma_{j}$ is the $j$ th singular value of matrix $Y$ and $\varepsilon=10^{-16}$ is to avoid dividing by zero. The values of the parameters $\tilde{\delta}$ and $C$ are related to the noise level of the speckle noise. We determine the best values of $\tilde{\delta}$ and $C$ such that the PSNR value of such a restored image with respect to such an original image is the smallest. In the ADMM for the low-rank approximation problem (3.1), we set the parameter $\mu=1.05$. According to Theorem 1 in [37], we know that the ADMM converges for any choice of the penalty parameter. However, this parameter does influence the speed of the algorithms. In experiments, we choose the best penalty parameter for a satisfactory speed. In the ADMM for the low-rank approximation problem (3.1), we choose the noisy patch group as the initialization. In addition, the iteration number of Newton's method is equal to 5 in the $X$-subproblem. We terminate the iterations for the proposed method when the maximum number of outer iterations $M=20$ has been carried out or the relative error between two successive iterations satisfies

$$
\frac{\left\|u^{k+1}-u^{k}\right\|_{2}}{\left\|u^{k+1}\right\|_{2}}<10^{-3} .
$$

For TV [12], HTV [16] and TGV [17], the quality of the restored images is highly dependent on the regularization parameters. We use the regularization parameter setting and the initialization following the original papers. For the BM3D method in [29], we apply the optimal regularization parameter for the highest PSNR of the restored image among all tested values of the regularization parameter. The DnCNN method in [35] is a state-of-the-art denoising algorithm based on deep learning for additive white Gaussian noise reduction. Since the DnCNN model is capable of handling Gaussian denoising with unknown noise level, the DnCNN method can be employed to reduce the noise in the ultrasound images $g=u+\sqrt{u} \eta$, where $\eta$ is a zero-mean Gaussian noise with standard deviation $\delta$. For the DnCNN method, we use the implementation function DnCNN and the default parameter settings from Matlab. 


\subsection{Experiments on simulated images}

In this section, we use eight gray-scale test images of size $256 \times 256$ for our experiments. The original images $u$ are shown in Fig. 3. The noisy images $g$ are generated by the degradation model (1.1), where the noise level is $\delta=1,2,3$, respectively.

To display how the patch size, step length between the consecutive key patches, nonlocal window size and number of patches in a group impact the performance of the proposed method, we carry out some experiments to search for the optimal parameters. In the first test, we use the first three images in Fig. 3 corrupted by the noise with the noise level $\delta=1$. In Fig. 4 , we plot the PSNR value by the proposed method as a function with respect to the patch size, step length between the consecutive key patches, nonlocal window size and number of patches in a group, respectively. From the results of Fig. 4, the basic parameter setting of the proposed patch-based method is as follows. The patch size is set as $6 \times 6$. We extract the key patch in every 4 pixels along with both horizontal and vertical directions. The nonlocal window size of searching similar patches is set as 30 . The number of similar patches in each group is chosen as $K=70$.

In Table 1, we show the values of PSNR and SSIM for the different test images by TV [12], HTV [16], TGV [17], BM3D [29], DnCNN [35] and the proposed method. The highest PSNR and SSIM values for each case are highlighted in bold. As can be seen from Table 1, our proposed method is averagely better than the other five methods for

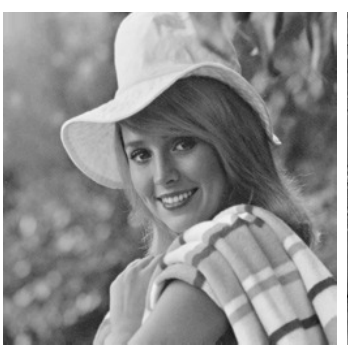

(a)

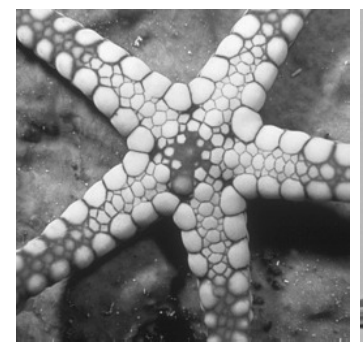

(e)

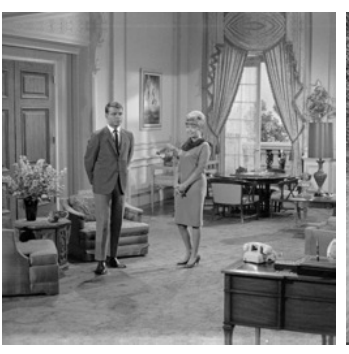

(b)

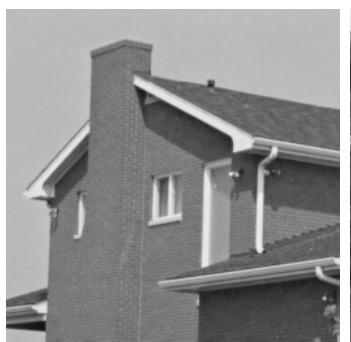

(f)

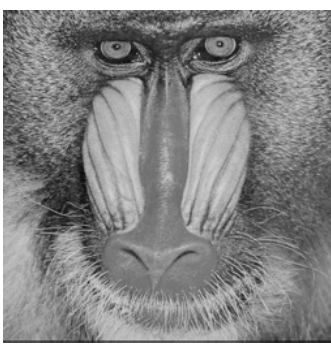

(c)

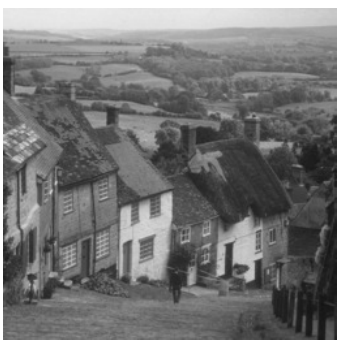

(g)

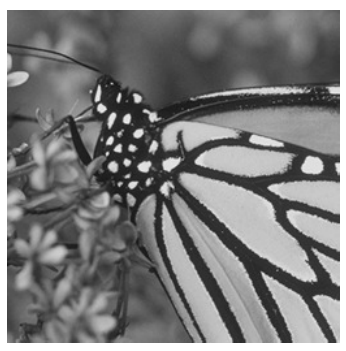

(d)

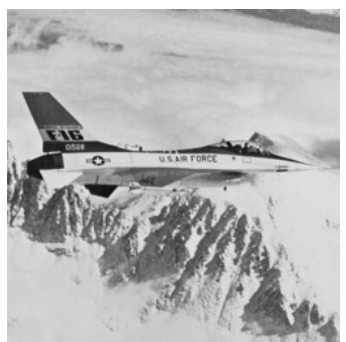

(h)

Figure 3: Test images. (a) Girl, (b) Couple, (c) Mandrill, (d) Monarch, (e) Starfish, (f) House, (g) Goldhill, (h) Airplane. 


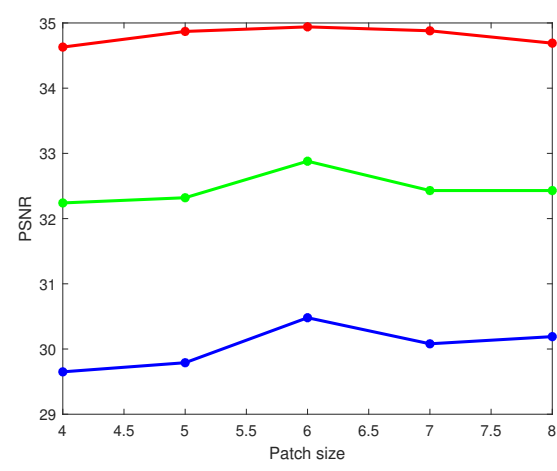

(a)

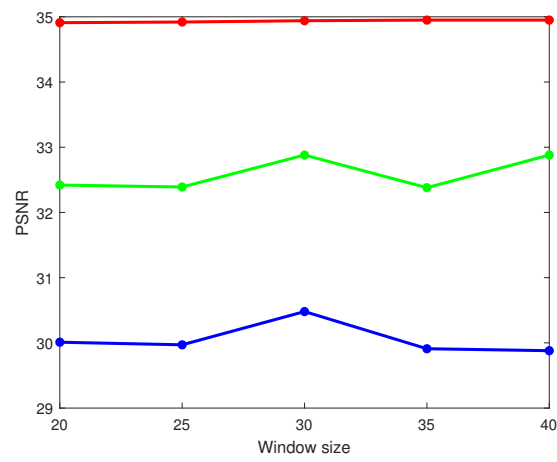

(c)

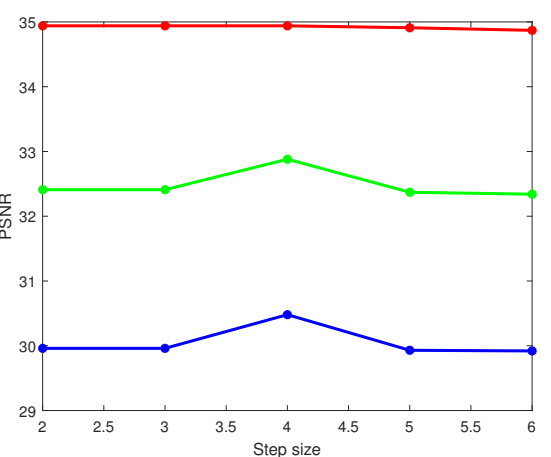

(b)

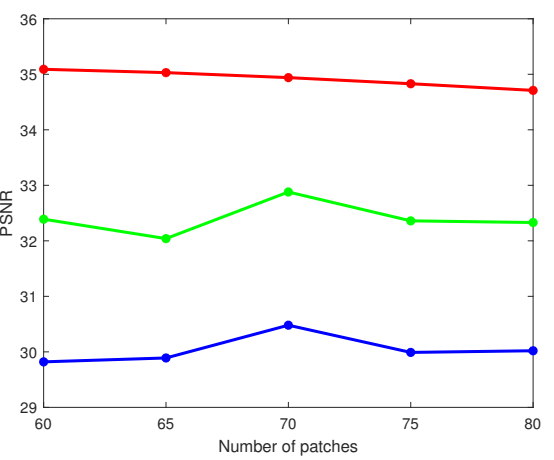

(d)

Figure 4: Parameter test. (a) Patch size, (b) Step size, (c) Window size, (d) Number of patches.

different noise levels with respect to PSNR and SSIM. Our proposed method achieves about $1.78 \mathrm{~dB}, 1.15 \mathrm{~dB}, 1.40 \mathrm{~dB}, 0.84 \mathrm{~dB}$ and $0.7 \mathrm{~dB}$ improvements in average over TV [12], HTV [16], TGV [17], BM3D [29] and DnCNN [35] respectively for the noise level $\delta=1$, while $1.74 \mathrm{~dB}, 1.16 \mathrm{~dB}, 1.43 \mathrm{~dB}, 0.46 \mathrm{~dB}$ and $0.42 \mathrm{~dB}$ improvements in average for the noise level $\delta=2$ and $1.59 \mathrm{~dB}, 0.96 \mathrm{~dB}, 1.22 \mathrm{~dB}, 0.29 \mathrm{~dB}$ and $0.11 \mathrm{~dB}$ improvements in average for the noise level $\delta=3$. It is not difficult to see from Table 1 that the deep learning method such as DnCNN [35] and the proposed method produce higher SSIM values in average than the patch-based method such as BM3D [29] and those methods without using patches such as TV [12], HTV [16] and TGV [17] for the three noise levels. Moreover, our proposed method performs better than DnCNN [35] with respect to the SSIM values on all test images for the noise level $\delta=1$. Our proposed method achieves higher SSIM values than DnCNN [35] for 2 out of 8 images under the noise levels $\delta=2$ and 3 out of 8 images under the noise levels $\delta=3$, respectively. In conclusion, compared with TV [12], HTV [16], TGV [17], BM3D [29] and DnCNN [35], our proposed method has achieved highly competitive restoration performance for different noise levels with respect to PSNR and SSIM. 
Table 1: Comparison of the performance of five speckle noise reduction methods.

\begin{tabular}{|c|c|c|c|c|c|c|}
\hline Mothed & TV & HTV & TGV & BM3D & DnCNN & Ours \\
\hline Image & PSNR/SSIM & PSNR/SSIM & PSNR/SSIM & PSNR/SSIM & PSNR/SSIM & PSNR/SSIM \\
\hline & & Noise level & $\delta=1$ & & & \\
\hline Girl & $33.08 / 0.8988$ & $34.35 / 0.9207$ & $33.68 / 0.9085$ & $33.73 / 0.8972$ & $34.29 / 0.9141$ & $34.94 / 0.9232$ \\
\hline Couple & $30.95 / 0.8739$ & $31.18 / 0.8774$ & $31.34 / 0.8833$ & $32.76 / 0.9169$ & $32.36 / 0.9051$ & $32.88 / 0.9177$ \\
\hline Mandrill & $28.76 / 0.8375$ & $29.29 / 0.8596$ & $28.89 / 0.8428$ & $30.02 / 0.8851$ & $29.61 / 0.8670$ & $30.48 / 0.8899$ \\
\hline Monarch & $32.33 / 0.9361$ & $33.26 / 0.9475$ & $32.80 / 0.9363$ & $33.28 / 0.9393$ & $33.22 / 0.9381$ & $34.05 / 0.9508$ \\
\hline Starfish & $30.41 / 0.8982$ & $31.46 / 0.9175$ & $31.30 / 0.9142$ & $31.24 / 0.9071$ & $31.78 / 0.9189$ & $32.56 / 0.9231$ \\
\hline House & $33.83 / 0.8781$ & $33.96 / 0.8756$ & $33.72 / 0.8792$ & $35.13 / 0.8869$ & $34.64 / 0.8866$ & $36.05 / 0.9116$ \\
\hline Goldhill & $31.76 / 0.8669$ & $32.12 / 0.8778$ & $32.20 / 0.8862$ & $32.91 / 0.8913$ & $32.89 / 0.9000$ & $33.07 / 0.9021$ \\
\hline Airplane & $31.74 / 0.9124$ & $32.27 / 0.9170$ & $32.01 / 0.9142$ & $31.43 / 0.8421$ & $32.71 / 0.9172$ & $33.05 / 0.9241$ \\
\hline Average & $31.61 / 0.8877$ & $32.24 / 0.8991$ & $31.99 / 0.8956$ & $32.56 / 0.8957$ & $32.69 / 0.9059$ & $33.39 / 0.9178$ \\
\hline & & Noise level & $\delta=2$ & & & \\
\hline Girl & $29.41 / 0.8313$ & $30.83 / 0.8709$ & $29.92 / 0.8253$ & $30.79 / 0.8411$ & $30.98 / 0.8490$ & $31.43 / 0.8743$ \\
\hline Couple & $27.30 / 0.7640$ & $27.50 / 0.7679$ & $27.80 / 0.7786$ & $29.11 / 0.8307$ & $28.86 / 0.8184$ & $29.15 / 0.8334$ \\
\hline Mandrill & $25.37 / 0.6703$ & $25.68 / 0.7245$ & $25.66 / 0.7011$ & $26.37 / 0.7400$ & $26.14 / 0.7290$ & $26.65 / 0.7585$ \\
\hline Monarch & $28.25 / 0.8802$ & $29.11 / 0.9009$ & $28.13 / 0.8418$ & $29.74 / 0.8975$ & $29.91 / 0.8868$ & $29.87 / \mathbf{0 . 9 1 1 4}$ \\
\hline Starfish & $26.34 / 0.7973$ & $27.39 / 0.8341$ & $27.30 / 0.8152$ & $27.89 / 0.8368$ & $28.50 / 0.8558$ & $28.63 / 0.8478$ \\
\hline House & $30.57 / 0.8358$ & $30.71 / 0.8319$ & $30.58 / 0.8358$ & $32.27 / 0.8182$ & $31.90 / 0.8337$ & $32.96 / 0.8547$ \\
\hline Goldhill & $28.42 / 0.7632$ & $28.70 / 0.7768$ & $28.64 / 0.7816$ & $29.50 / 0.7954$ & $29.70 / 0.8158$ & $29.62 / 0.7993$ \\
\hline Airplane & $28.00 / 0.8511$ & $28.36 / 0.8559$ & $28.13 / 0.8418$ & $28.24 / 0.7522$ & $29.23 / 0.8501$ & $29.30 / 0.8760$ \\
\hline Average & $27.96 / 0.7992$ & $28.54 / 0.8204$ & $28.27 / 0.8025$ & $29.24 / 0.8140$ & $29.28 / 0.8298$ & $29.70 / 0.8444$ \\
\hline & & Noise level & $\delta=3$ & & & \\
\hline Girl & $27.13 / 0.7782$ & $28.74 / 0.8309$ & $28.21 / 0.8116$ & $29.02 / 0.8203$ & $28.83 / 0.7473$ & $29.23 / 0.8238$ \\
\hline Couple & $25.16 / 0.6769$ & $25.78 / 0.6961$ & $25.73 / 0.6964$ & $26.12 / 0.7126$ & $26.88 / 0.7426$ & $26.18 / 0.7174$ \\
\hline Mandrill & $23.84 / 0.5583$ & $23.54 / 0.5264$ & $23.65 / 0.5396$ & $23.59 / 0.5117$ & $24.52 / 0.6179$ & $24.10 / 0.5683$ \\
\hline Monarch & $25.84 / 0.8308$ & $26.84 / 0.8596$ & $26.63 / 0.8517$ & $27.12 / 0.8600$ & $27.78 / 0.8412$ & $27.83 / 0.8697$ \\
\hline Starfish & $24.53 / 0.7298$ & $25.38 / 0.7711$ & $24.76 / 0.7423$ & $26.36 / 0.7880$ & $26.28 / 0.7947$ & $26.50 / 0.7883$ \\
\hline House & $28.42 / 0.8074$ & $28.77 / 0.8021$ & $28.46 / 0.7987$ & $31.16 / 0.8356$ & $29.89 / 0.7849$ & $31.24 / 0.8360$ \\
\hline Goldhill & $26.56 / 0.6864$ & $26.99 / 0.7116$ & $26.91 / 0.7113$ & 27.36/0.7131 & $27.80 / 0.7473$ & $27.70 / \mathbf{0 . 7 8 0 5}$ \\
\hline Airplane & $25.91 / 0.8030$ & $26.39 / 0.8091$ & $25.96 / 0.8053$ & $27.06 / 0.8263$ & $27.18 / 0.7927$ & $27.31 / 0.8357$ \\
\hline Average & $25.92 / 0.7339$ & $26.55 / 0.7509$ & $26.29 / 0.7446$ & $27.22 / 0.7584$ & $27.40 / 0.7586$ & $27.51 / 0.7774$ \\
\hline
\end{tabular}

In Figs. 5 and 6, we show the speckle noise reduction ability of our proposed method over the other five methods by recovering the images "Girl" and "Couple" for the noise level $\delta=1$. We show the restored images by TV [12], HTV [16], TGV [17], BM3D [29], DnCNN [35] and our proposed method in Figs. 5(c)-(h) and 6(c)-(h) respectively. We observe from the figures that our proposed method yields more satisfactory results in terms of visual performance. Our proposed method is able to avoid the artifact effect and achieves effective preservation of details. As can be seen from Table 1, our proposed method achieves the highest PSNR and SSIM values among the six speckle noise reduction methods. For a better comparison, we enlarge some details of the restored images and display them in the bottom left corner. As can be seen from the zoomed part, the restored images in Figs. 5(h) and 6(h) by our proposed method look more natural, and they match the original image in Figs. 5(a) and 6(a) respectively in a better way. 


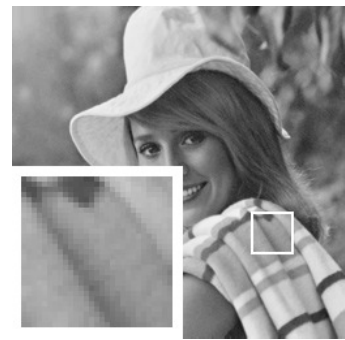

(a)

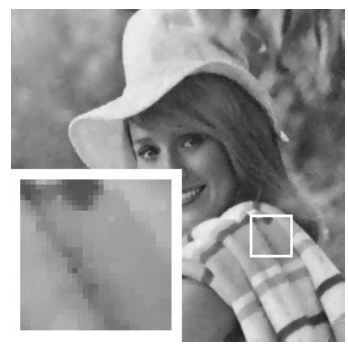

(e)

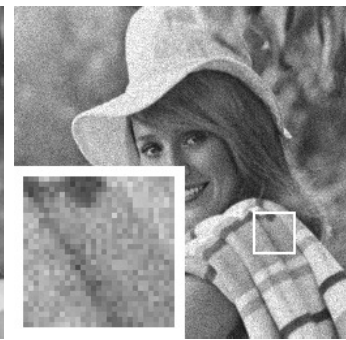

(b)

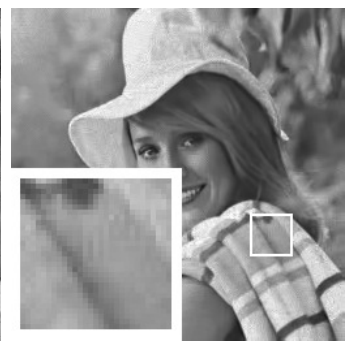

(f)

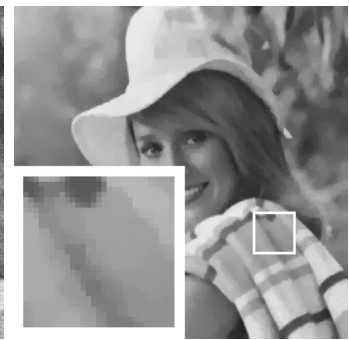

(c)

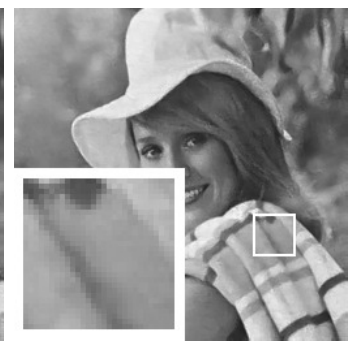

(g)

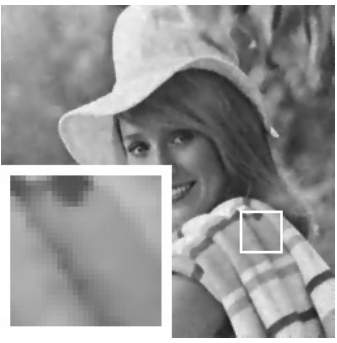

(d)

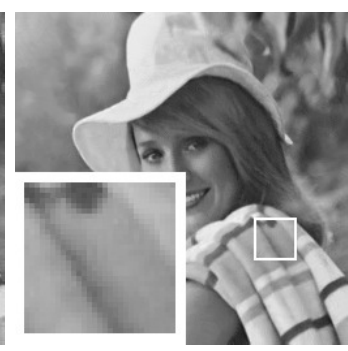

(h)

Figure 5: Restoration results for the "Girl" image. (a) True, (b) Noisy, (c) TV, (d) HTV, (e) GTV, (f) BM3D, (g) DnCNN, (h) Ours.

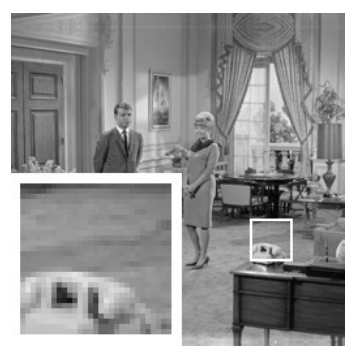

(a)

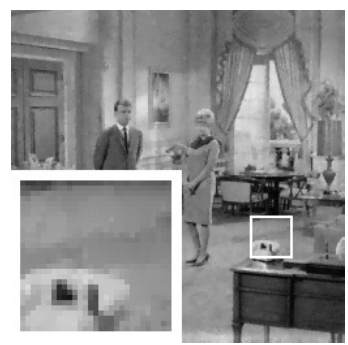

(e)

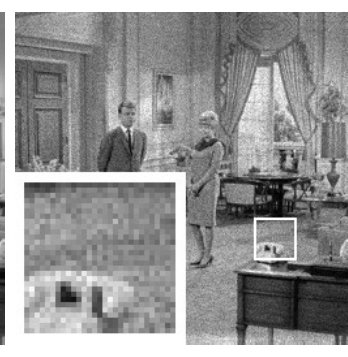

(b)

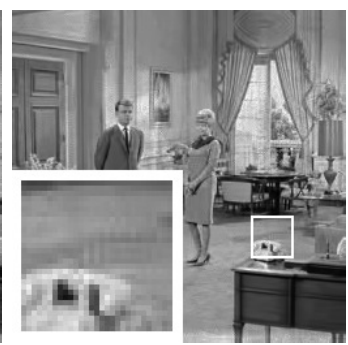

(f)

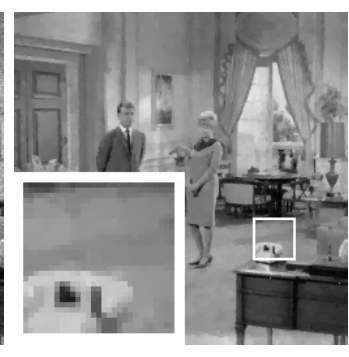

(c)

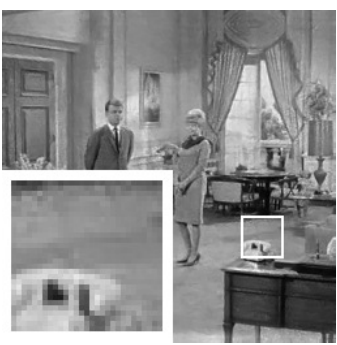

(g)

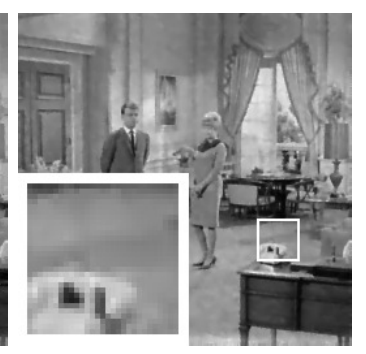

(d)

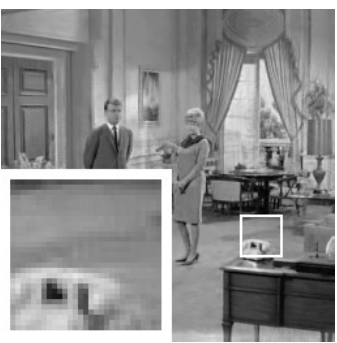

(h)

Figure 6: Restoration results for the "Couple" image. (a) True (b) Noisy, (c) TV, (d) HTV, (e) GTV, (f) BM3D, (g) DnCNN, (h) Ours. 


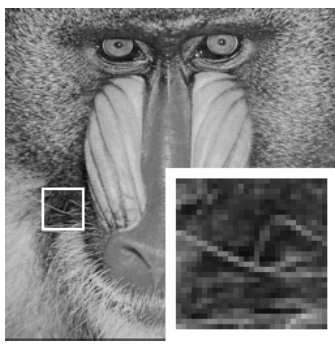

(a)

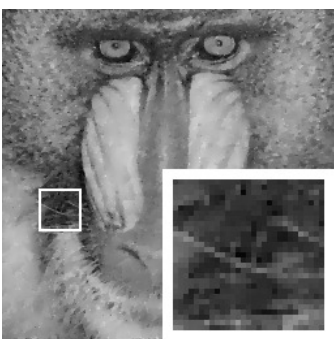

(e)

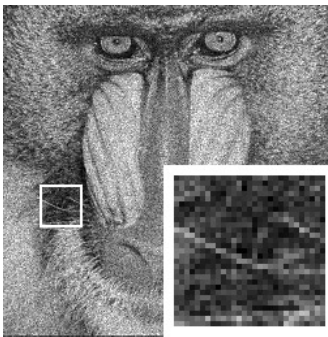

(b)

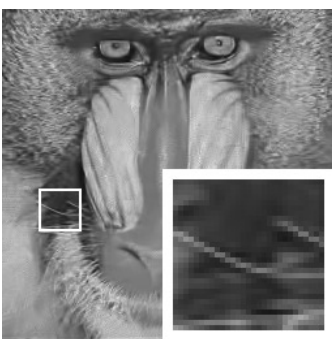

(f)

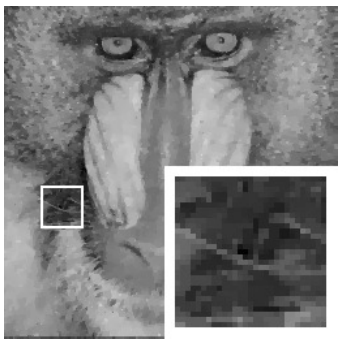

(c)

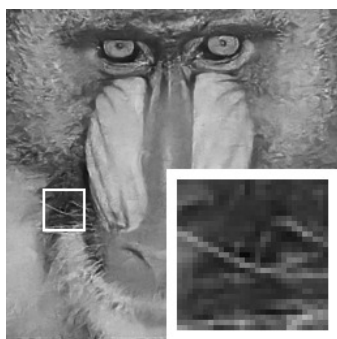

(g)

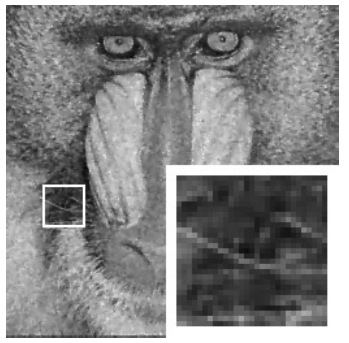

(d)

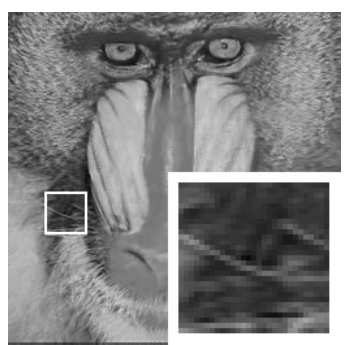

(h)

Figure 7: Restoration results for the "Mandrill" image. (a) True, (b) Noisy, (c) TV, (d) HTV, (e) GTV, (f) BM3D, (g) DnCNN, (h) Ours.

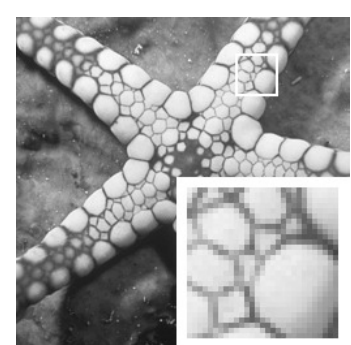

(a)

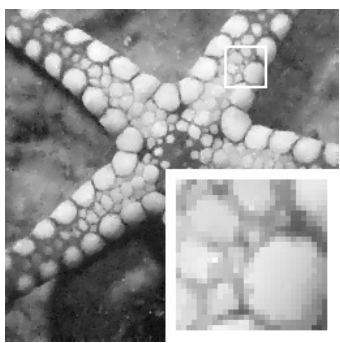

(e)

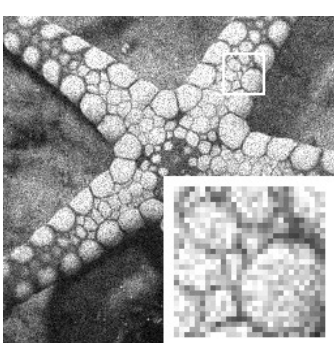

(b)

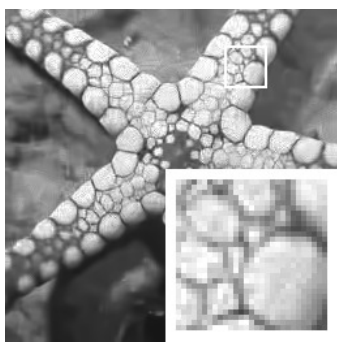

(f)

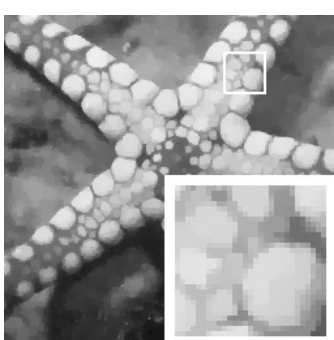

(c)

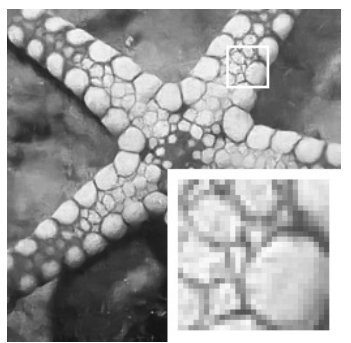

(g)

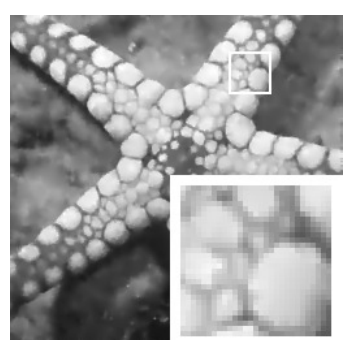

(d)

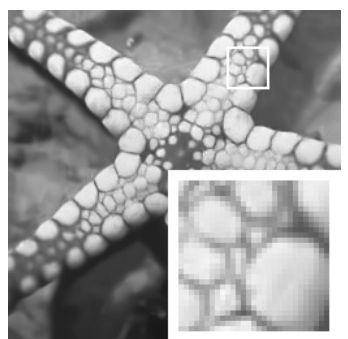

(h)

Figure 8: Restoration results for the "Starfish" image. (a) True, (b) Noisy, (c) TV, (d) HTV, (e) GTV, (f) BM3D, (g) DnCNN, (h) Ours. 
We give the restoration results for the images "Mandrill" and "Starfish" under the noise level $\delta=2$ in Figs. 7 and 8. The restored images by TV [12], HTV [16], TGV [17], BM3D [29], DnCNN [35] and our proposed method are displayed in Figs. 7(c)-(h) and 8(c)-(h) respectively. It is clear from Figs. 7 and 8 that our proposed method outperforms the other five methods. The images recovered by the proposed method have more details than those recovered by TV [12], HTV [16], TGV [17], BM3D [29] and DnCNN [35], and at the same time, the edges can be preserved as well. Table 1 shows that compared with the other four methods, DnCNN [35] and our proposed method perform better for restoring images with respect to the PSNR and SSIM values. In order to gain further insight into the performance of our proposed method, the zoomed-in parts of the restored images are shown in the bottom right corner. It is easy to see that our proposed method exhibits sharp edges and preserves detailed features in the restored images while removing the speckle noise well.

In Figs. 9 and 10, we compare the restored images produced by our proposed method with those produced by TV [12], HTV [16], TGV [17], BM3D [29] and DnCNN [35] for the images "House" and "Airplane". The original images in Figs. 9(a) and 10(a) are corrupted by the speckle noise with the noise level $\delta=3$ and the noisy images are displayed in Figs. 9(b) and 10(b). In Figs. 9(c)-(h) and 10(c)-(h), we show the restored images by TV [12], HTV [16], TGV [17], BM3D [29], DnCNN [35] and our proposed method. It can be seen from these figures that the performance of our proposed method is quite good and is comparable to the other five restoration methods. From Table 1, we know that our proposed method has higher PSNR and SSIM values than the other five methods. For better visualization, we zoom in on small parts of the recovered images and display them in the lower right corner. It is obvious that our method obtains better results than the other five methods in terms of visual quality.

\subsection{Experiments on real ultrasound images}

In this section, we test the performance of the proposed method on four real ultrasound images and compare with TV [12], HTV [16], TGV [17], BM3D [29] and DnCNN [35]. Four real noisy ultrasound images are displayed in Figs. 11(a), 12(a), 13(a) and 14(a) respectively. The restored images by TV [12], HTV [16], TGV [17], BM3D [29], DnCNN [35] and the proposed method are shown in Figs. 11(b)-(g), 12(b)-(g), 13(b)-(g) and 14(b)-(g), respectively. It is not difficult to see from the figures that the five methods such as TV [12], HTV [16], TGV [17], BM3D [29] and the proposed method have speckle noise reduction ability for real ultrasound images. The DnCNN method in [35] almost doesn't detect the noise in real ultrasound images. We don't see obvious noise reduction in Figs. 11(f), 12(f), 13(f) and 14(f) for real ultrasound images. The enlarged parts of the recovered ultrasound images are given for better visual comparison. From the enlarged parts in Figs. 11(b)14(b), the staircase effect can be detected in the restored images by TV [12]. The restored images by HTV [16], TGV [17], BM3D [29] and the proposed method are much better than the restored images by TV [12] in terms of the staircase effect. Visually, BM3D [29] 


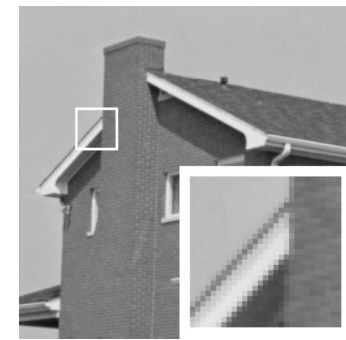

(a)

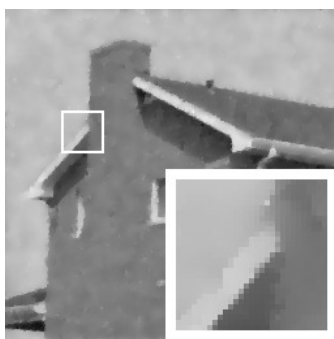

(e)

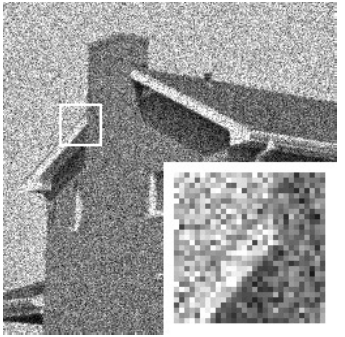

(b)

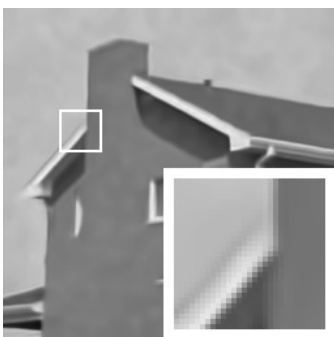

(f)

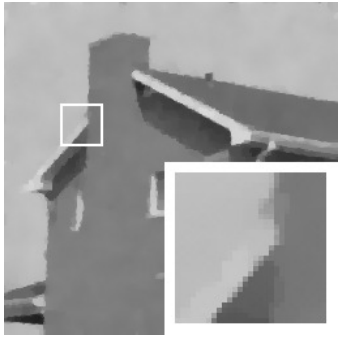

(c)

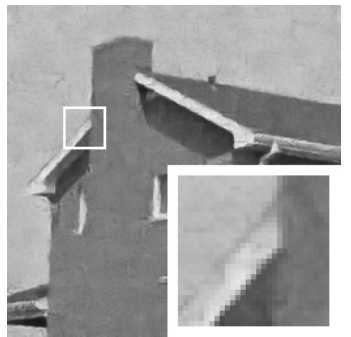

(g)

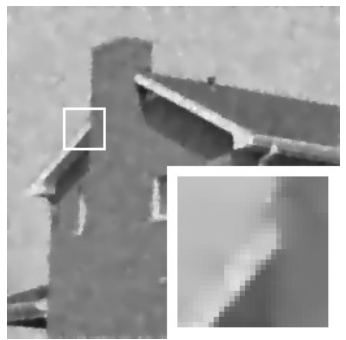

(d)

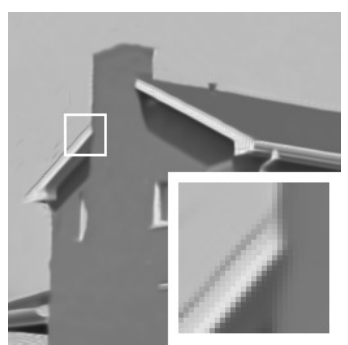

(h)

Figure 9: Restoration results for the "House" image. (a) True, (b) Noisy, (c) TV, (d) HTV, (e) GTV, (f) BM3D, (g) DnCNN, (h) Ours.

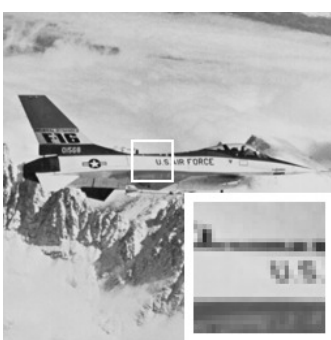

(a)

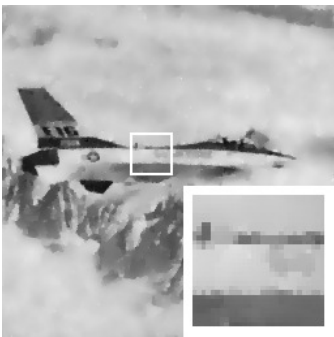

(e)

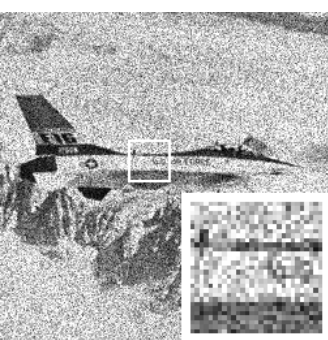

(b)

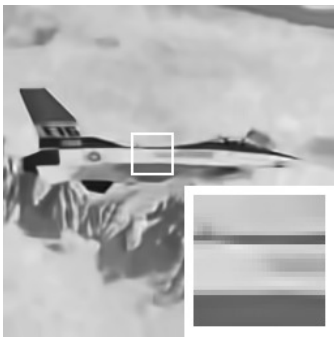

(f)

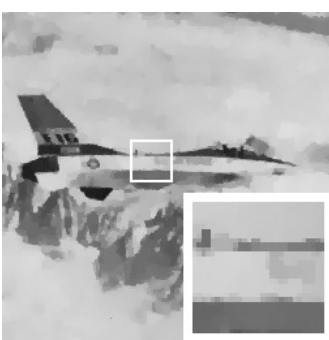

(c)

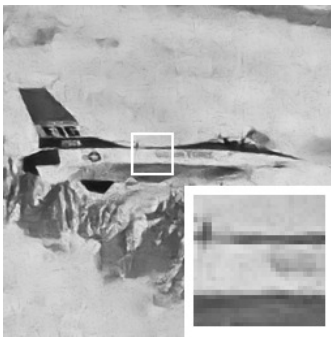

(g)

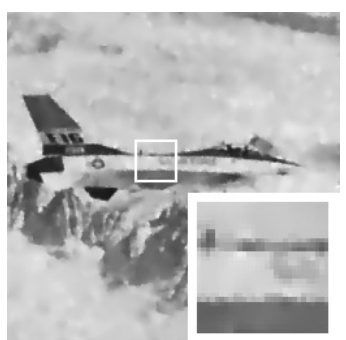

(d)

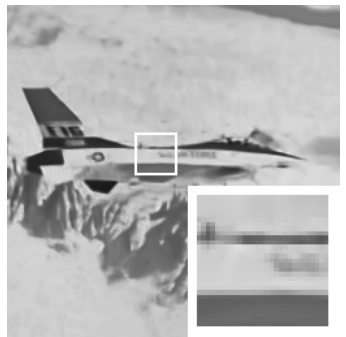

(h)

Figure 10: Restoration results for the "Airplane" image. (a) True, (b) Noisy, (c) TV, (d) HTV, (e) GTV, (f) BM3D, (g) DnCNN, (h) Ours. 


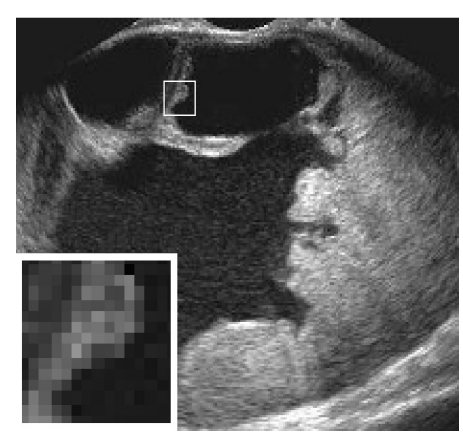

(a)

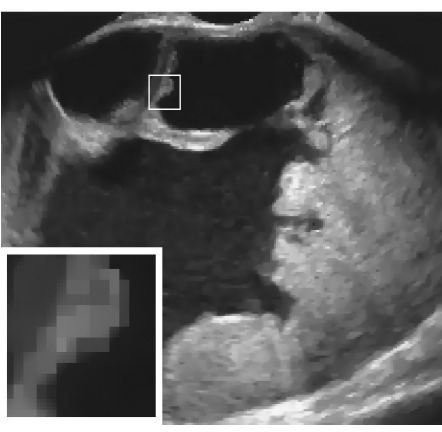

(b)

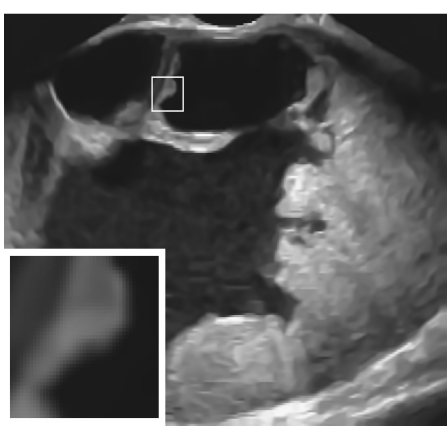

(e)

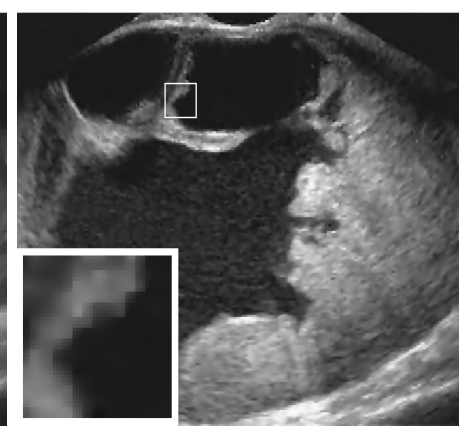

(c)

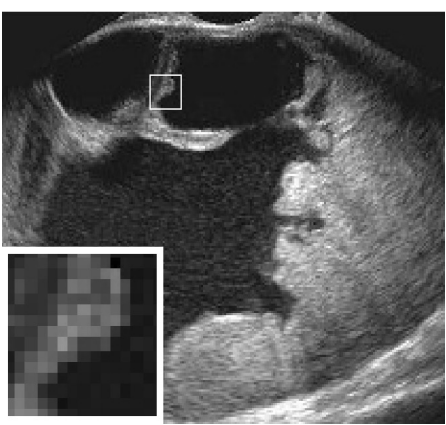

(f)

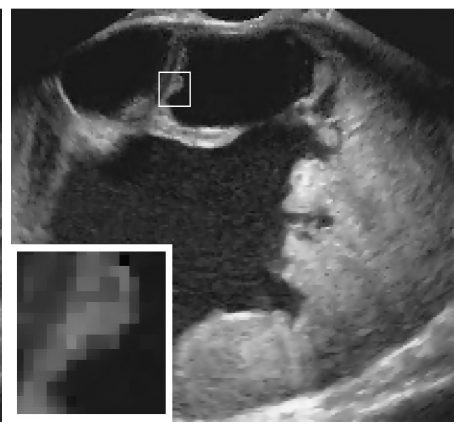

(d)

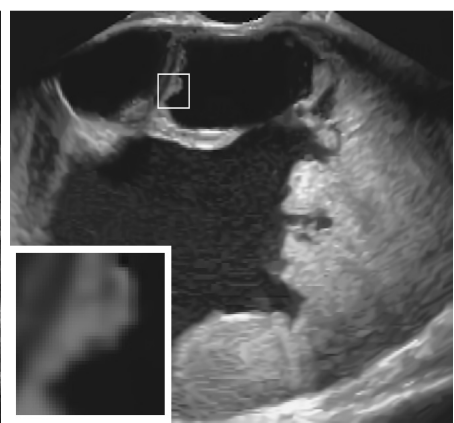

$(g)$

Figure 11: Restoration results. (a) Real ultrasound image, (b) TV, (c) HTV, (d) GTV, (e) BM3D, (f) DnCNN, (g) Ours.

has a strong detail-preserving ability. However, BM3D [29] cannot suppress the speckle noise adequately and may introduce some undesired artifacts. Compared with TV [12], HTV [16], TGV [17], BM3D [29] and DnCNN [35], the proposed method can provide better restoration results with effective speckle noise reduction and without artifacts. At the same time, detailed features in the restored images by the proposed method can be preserved very well. 


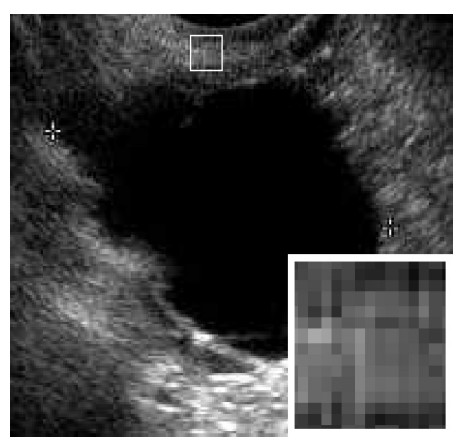

(a)

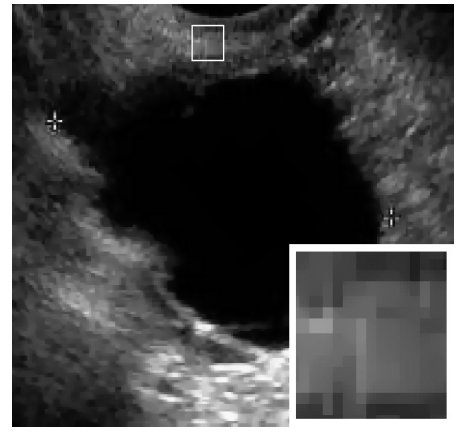

(b)

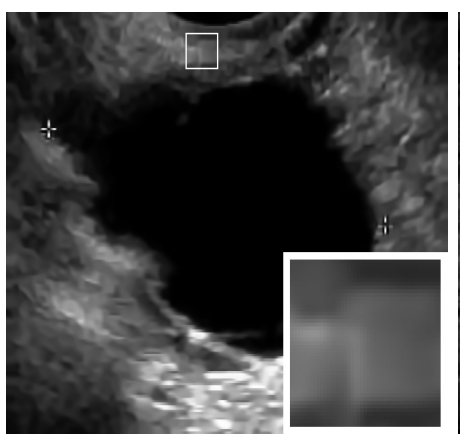

(e)

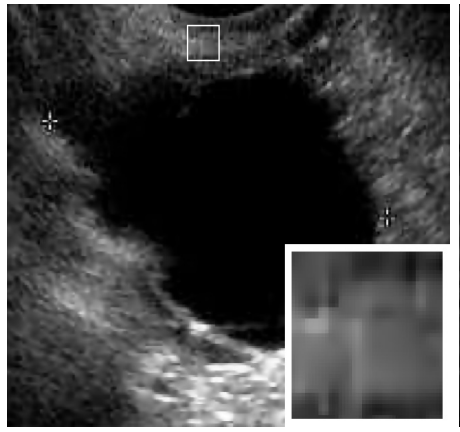

(c)

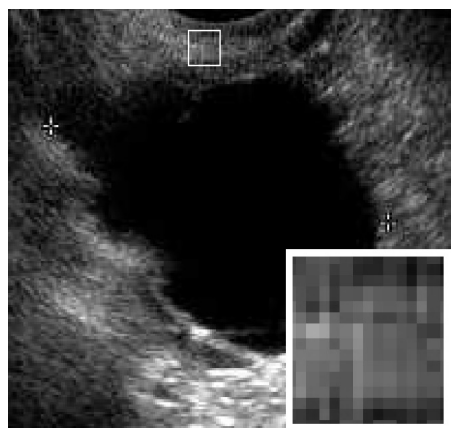

(f)

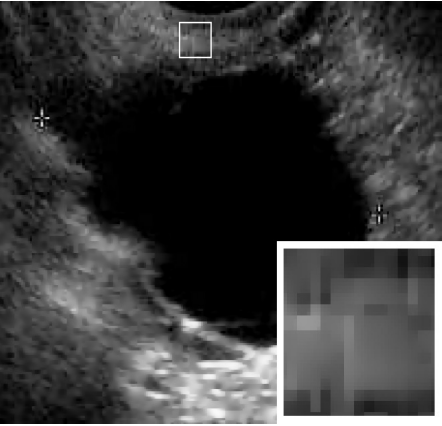

(d)

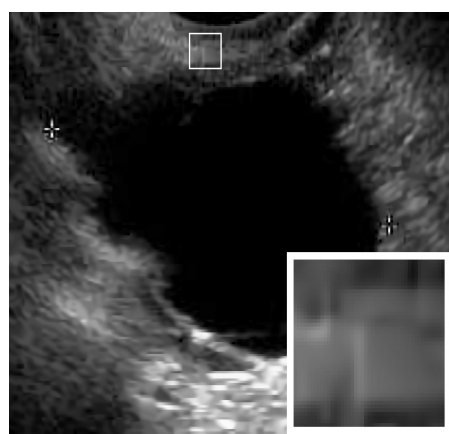

(g)

Figure 12: Restored results. (a) Real ultrasound image, (b) TV, (c) HTV, (d) GTV, (e) BM3D, (f) DnCNN, (g) Ours.

\section{Conclusions}

We investigate a patch-based low-rank approach for reducing the speckle noise in ultrasound images in this paper. Firstly, some similar patches are selected for each key patch to construct a patch group. Then a nonconvex variational model is proposed by 


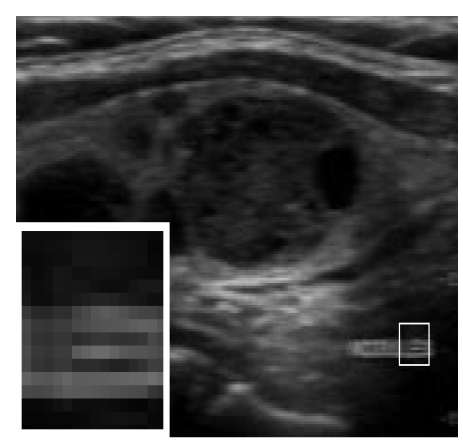

(a)

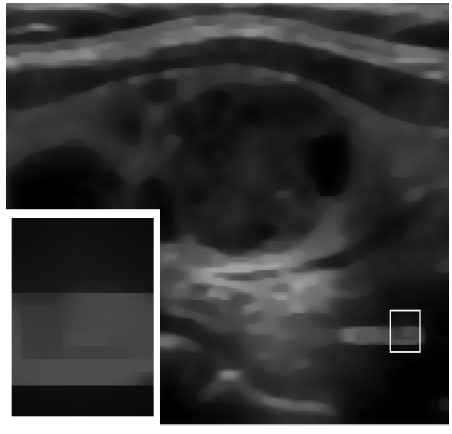

(b)

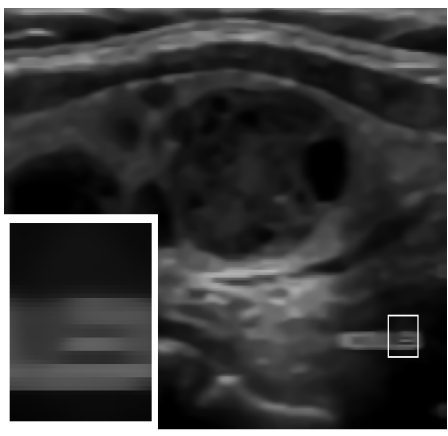

(e)

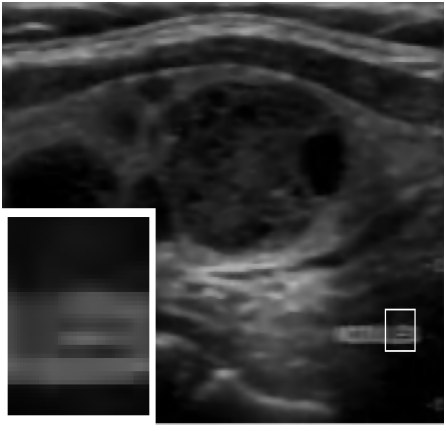

(c)

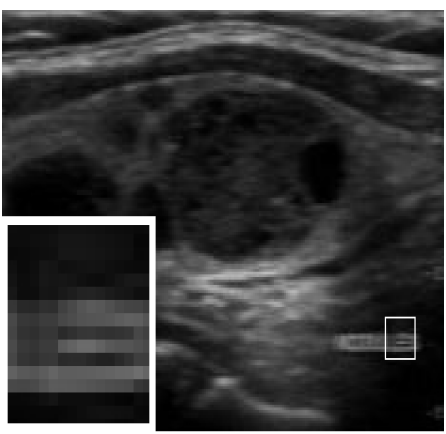

(f)

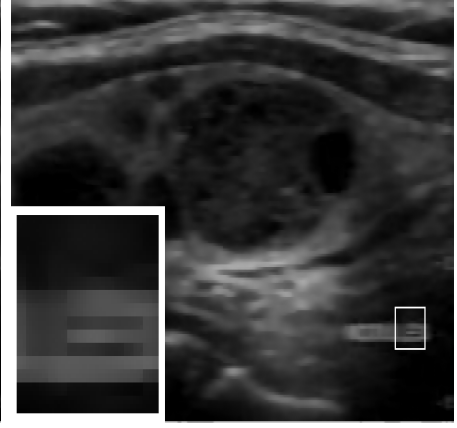

(d)

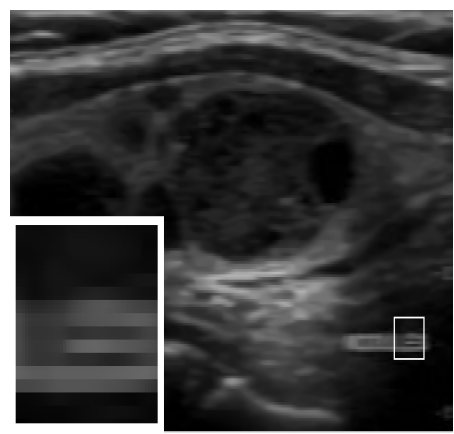

(g)

Figure 13: Restored results. (a) Real ultrasound image, (b) TV, (c) HTV, (d) GTV, (e) BM3D, (f) DnCNN, (g) Ours.

using the weighted nuclear norm as a regularizer for the patch group. We employ the effective ADMM method for the approximate solution of the proposed nonconvex model. Finally, all the approximate patches are returned to their original locations to generate the noise reduction ultrasound images. Experimental results on both simulated and real ultrasound images are given to demonstrate that the proposed method outperforms some 


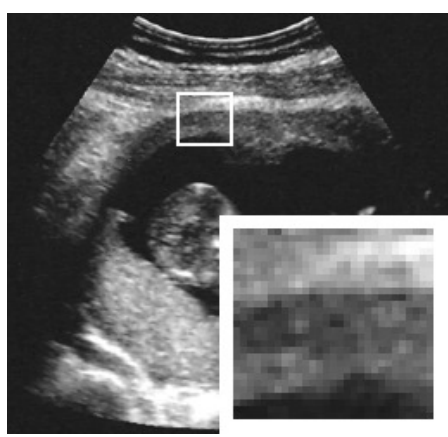

(a)

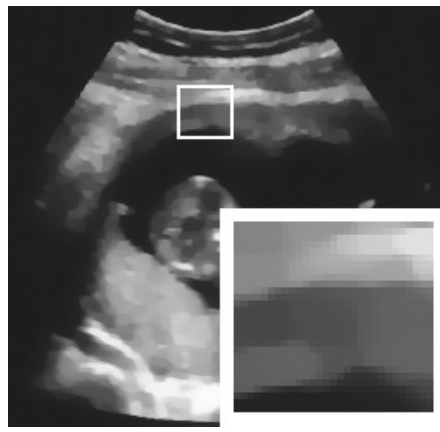

(b)

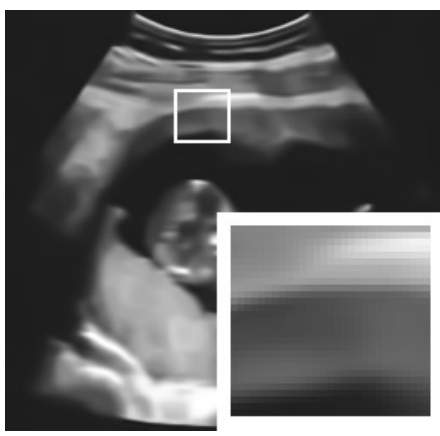

(e)

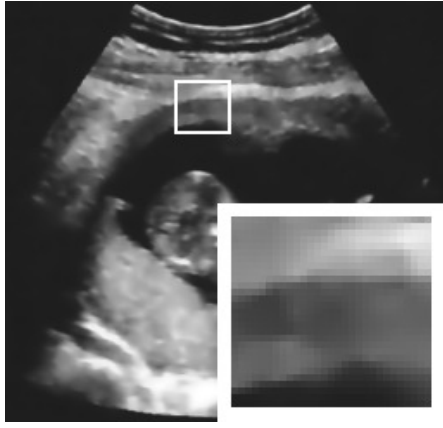

(c)

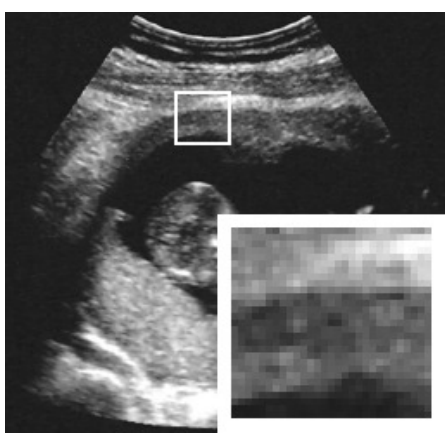

(f)

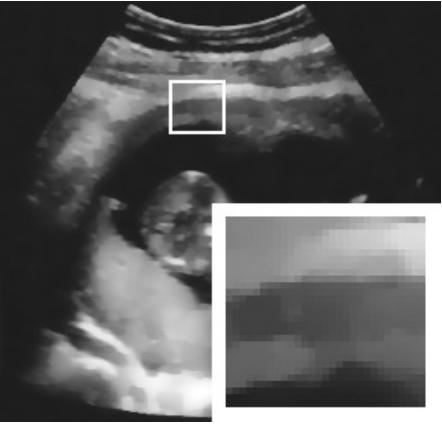

(d)

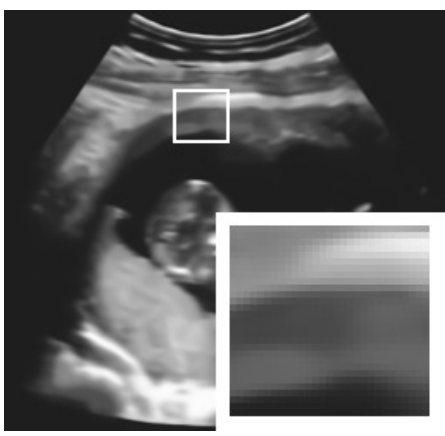

(g)

Figure 14: Restored results. (a) Real ultrasound image, (b) TV, (c) HTV, (d) GTV, (e) BM3D, (f) DnCNN, (g) Ours.

existing state-of-the-art methods such as TV [12], HTV [16], TGV [17] BM3D [29] and DnCNN [35] in terms of visual quality, PSNR and SSIM measures.

The main running time consuming in the proposed method is the finding of similar patches and the computations of SVD. The SVD computations needed in the WNNM problem are very expensive, which bring down the efficiency of the proposed method. 
Our proposed method is more time-consuming than TV [12], HTV [16], TGV [17], BM3D [29] and DnCNN [35]; however, this drawback is compensated by its outstanding performance. In the next step, we consider how to speed up the proposed method by converting the code to $\mathrm{C} / \mathrm{C}++$ and GPU implementation with parallelization. On the other hand, we consider employing the fast randomized singular value thresholding method to reduce the computational time of the WNNM problem without losing accuracy and hurting convergence behavior. In the future work, we will investigate the acceleration of the proposed method.

\section{Acknowledgements}

The authors would like to express their great thankfulness to the referees for much constructive, detailed and helpful advice regarding revising this manuscript. This work was supported by NSF of Jiangsu Province (No. BK20181483), NSFC (Nos. 11671002, 11701079, 61731009), the Fundamental Research Funds for the Central Universities, and Science and Technology Commission of Shanghai Municipality (Nos. 19JC1420102, 18dz2271000), Hai Yan project, Lianyungang 521 project and NSF of HHIT (No. Z2017004).

\section{References}

[1] K. DJEMAL, Speckle reduction in ultrasound images by minimization of total variation, in IEEE International Conference on Image Processing, 2005.

[2] H. Lim AND T. M. Williams, A non-standard anisotropic diffusion for speckle noise removal, J. Syst. Cybernetics Inform., 5(2) (2007), pp. 12-17.

[3] K. KRISSIAN, R. KIKINIS, C. F. WESTIN, AND K. G. Vosburgh, Speckle-constrained filtering of ultrasound images, in Proceedings of the IEEE Conference on Computer Vision and Pattern Recognition, 2005.

[4] A. GARG AND V. KHANDELWAL, Speckle noise reduction in medical ultrasound images using coefficient of dispersion, in International Conference on Signal Processing \& Communication, 2016.

[5] M. HaCini, F. Hachouf, AND K. DJEMAL, A new speckle filtering method for ultrasound images based on a weighted multiplicative total variation, Signal Process., 103 (2014), pp. 214-229.

[6] M. G. Shama, T. Z. HuAnG, J. LiU, AND S. WANG, A convex total generalized variation regularized model for multiplicative noise and blur removal, Appl. Math. Comput., 276 (2016), pp. 109-121.

[7] C. LIU, M. NG, AND T. ZENG, Weighted variational model for selective image segmentation with application to medical images, Pattern Recognition, 76 (2018), pp. 367-379.

[8] N. Chumchob AND I. PRAKIT, An improved variational model and its numerical solutions for speckle noise removal from real ultrasound images, J. Comput. Math., 37(2) (2019), pp. 201-239.

[9] F. Fontes, G. Barroso, P. Coupe, AND P. Hellier, Real time ultrasound image denoising, J. Real Time Image Process., 6(1) (2011), pp. 15-22. 
[10] Celia A. Z. BARCelos And Luciana E. S. R. Vieira, Ultrasound speckle noise reduction via an adaptive edge-controlled variational method, in IEEE International Conference on Systems, Man and Cybernetics (SMC), 2014.

[11] C. P. LoizOU AND C. S. PATTICHIS, Despeckle Filtering Algorithms and Software for Ultrasound Imaging, Morgan \& Claypool, 2015.

[12] Z. M. JIN AND X. P. YANG, A variational model to remove the multiplicative noise in ultrasound images, J. Math. Imag. Vision, 39(1) (2011), pp. 62-74.

[13] H. JIE AND X. P. YANG, Fast reduction of speckle noise in real ultrasound images, Signal Process., 93(4) (2013), pp. 684-694.

[14] Y. L. WU AND X. C. FENG, Speckle noise reduction via nonconvex high total variation approach, Math. Problems Eng., 2015(2) (2015), pp. 1-11.

[15] M. KANG, M. KANG, AND M. JUNG, Total generalized variation based denoising models for ultrasound images, J. Sci. Comput., 72(1) (2017), pp. 172-197.

[16] S. WAnG, T. Z. HuAng, X. L. ZhaO, J. J. MeI, AND J. HuAng, Speckle noise removal in ultrasound images by first- and second-order total variation, Numer. Algorithms, 78 (2018), pp. 513-533.

[17] J. J. MeI, T. Z. HuAnG, S. WANG, AND X. L. ZHAO, Second order total generalized variation for speckle reduction in ultrasound images, J. Franklin Institute, 355 (2018), pp. 574-595.

[18] B. A. ABRAHIM AND Y. KADAH, Speckle noise reduction method combining total variation and wavelet shrinkage for clinical ultrasound imaging, in IEEE 1st Middle East Conference on Biomedical Engineering, pages 80-83, 2011.

[19] Y. XIAO, T. ZENG, J. YU, AND M. K. NG, Restoration of images corrupted by mixed Gaussianimpulse noise via l1-l0 minimization, Pattern Recognition, 44(8) (2011), pp. 1708-1720.

[20] Y. M. HuANG, L. MoISAN, M. K. NG, AND T. Y. ZENG, Multiplicative noise removal via a learned dictionary, IEEE Trans. Image Process., 21(11) (2012), pp. 4534-4543.

[21] L. MA, L. MOISAN, J. YU, AND T. ZENG, A dictionary learning approach for poisson image deblurring, IEEE Trans. Medical Imag., 32(7) (2013), pp. 1277-1289.

[22] L. Sun, J. CHEN, D. ZENG, AND X. DING, A novel nonlocal MRI reconstruction algorithm with patch-based low rank regularization, in IEEE Global Conference on Signal and Information Processing (GlobalSIP), 2015.

[23] H. HU, J. FROMENT, AND Q. LIU, A note on patch-based low-rank minimization for fast image denoising, J. Visual Commun. Image Representation, 50 (2018), pp. 100-110.

[24] K. DABOV, A. FOI, V. KATKOVNIK, AND K. EGIAZARIAN, Image denoising by sparse $3 d$ transform-domain collaborative filtering, IEEE Trans. Image Process., 16(8) (2007), pp. 20802095.

[25] S. GU, L. ZHANG, W. ZUO, AND X. FENG, Weighted nuclear norm minimization with application to image denoising, in Proceedings of the IEEE Conference on Computer Vision and Pattern Recognition, pages 2862-2869, 2014.

[26] L. MA, L. XU, AND T. ZENG, Low rank prior and total variation regularization for image deblurring, J. Sci. Comput., 70 (2017), pp. 1336-135721.

[27] D. GUAN, D. XIANG, X. TANG, AND G. KUANG, SAR image despeckling based on nonlocal low-rank regularization, IEEE Transactions on Geoscience and Remote Sensing, pages 1-18, 2018.

[28] G. KIM, J. CHO, AND M. KANG, Cauchy noise removal by weighted nuclear norm minimization, J. Sci. Comput., 83(15) (2020), pp. 1-21.

[29] Y. GAN, E. ANGelini, A. LAINE, AND C. HENDON, BM3D-based ultrasound image denoising via brushlet thresholding, in IEEE 12th International Symposium on Biomedical Imaging, 
pages 667-670, 2015.

[30] X. LV AND F. LI, An iterative decoupled method with weighted nuclear norm minimization for image restoration, Int. J. Comput. Math., 15(3) (2020), pp. 602-623.

[31] X. YANG AND Y. HUANG, A modulus iteration method for SPSD linear complementarity problem arising in image retinex, Adv. Appl. Math. Mech., 12(2) (2020), pp. 579-598.

[32] S. Boyd, N. PARIKh, E. ChU, B. Peleato, AND J. ECKSTEIN, Distributed optimization and statistical learning via the alternating direction method of multipliers, Foundations and Trends in Machine Learning, 3(1) (2011), pp. 1-122.

[33] T. WU AND J. SHAO, Non-convex and convex coupling image segmentation via TG $p$ V regularization and thresholding, Adv. Appl. Math. Mech., 12(3) (2020), pp. 849-878.

[34] T. WU, Z. PANG, Y. WANG, AND Y. YANG, CS-MRI reconstruction based on the constrained TGV-shearlet scheme, Int. J. Numer. Anal. Model., 17 (2020), pp. 316-331.

[35] K. ZhANG, W. ZuO, Y. CHEN, D. Meng, AND L. ZHANG, Beyond a gaussian denoiser: Residual learning of deep CNN for image denoising, IEEE Trans. Image Process., 26(7) (2017), pp. 3142-3155.

[36] Z. WANG, A. C. BOviK, H. R. SHeIKH, AND E. P. SimONCELli, Image quality assessment: from error visibility to structural similarity, IEEE Trans. Image Process., 13(4) (2004), pp. 600612.

[37] M. A. T. FIgUEIREDO AND J. M. BIOUCAS-DiAs, Restoration of poissonian images using alternating direction optimization, IEEE Trans. Image Process., 19(12) (2010), pp. 3133-3145. 